\begin{tabular}{|c|c|c|}
\hline Beitr. Ent. & Keltern & ISSN 0005-805X \\
\hline $\mathbf{6 2}(2012) 2$ & S. $263-286$ & 20.12 .2012 \\
\hline
\end{tabular}

\title{
Prof. Dr. Joachim Oehlke - 75 Jahre
}

Mit 9 Figuren und 2 Tabellen

\section{Frank Menzel, Andreas Taeger, Ortrud Taeger, Jens Möller, Stephan M. Blank, Christian Kutzscher und Lutz Behne}

Am 19. Dezember 2011 beging der Eberswalder Hymenopterologe Prof. Dr. Joachim Oehlke seinen 75. Geburtstag. Die vielseitigen Aktivitäten und Leistungen des Jubilars lassen sich in einer Festschrift kaum umfassend würdigen. Darum kann man viele Bereiche, in denen Joachim Oehlke Hervorragendes und Bleibendes geleistet hat, sicher nur unvollständig, zu kurz oder nicht in der richtigen Reihenfolge darstellen. Mitunter haben die Autoren auch einfach die ganze Bandbreite seines Schaffens nicht überblicken können, weil sie lediglich einen Bruchteil

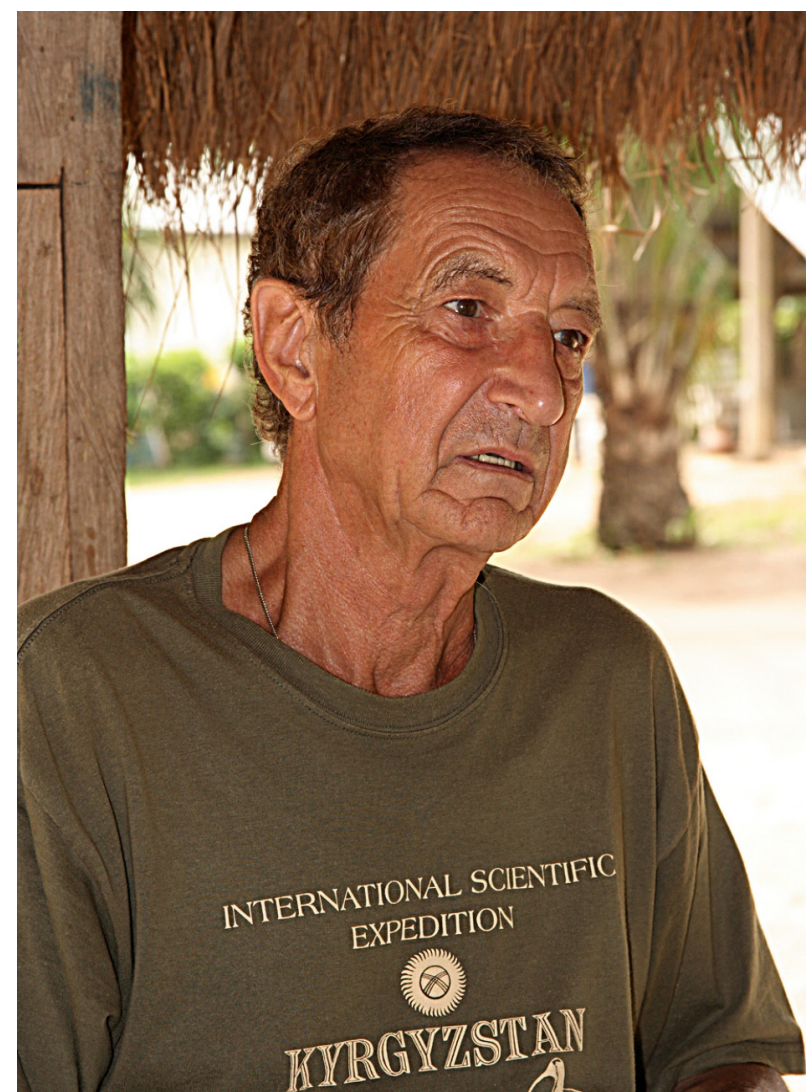

Fig. 1: Prof. Dr. Joachim Oehlke im April 2008 in Togo. Foto: F. Menzel. 
seines ausgefüllten Lebens als Insekten-, Fisch-, Amphibien- und Reptilienkundler; Züchter, zoologischer Präparator und Hochschullehrer; Naturschützer und Lehrmittelproduzent oder als Sportler und gesellschaftlich engagierte Persönlichkeit begleitet haben.

Hermann Hans Joachim Oehlke (von seinen Kollegen und Freunden liebevoll „Achim“ genannt) wurde am 19. Dezember 1936 in Breslau (Schlesien, heute Wrocław) geboren. Seine Mutter Johanna Oehlke, geborene Wilde (1900-1948), war als Verkäuferin und als Erzieherin in einem Kinderheim tätig. Sein Vater Karl Oehlke (1898-1974) übte den Beruf eines Mechanikers für Fahrräder und Nähmaschinen aus und arbeitete seit 1948 als selbständiger Mechanikermeister. Aus der elterlichen Ehe ging auch noch der ältere Bruder Karl-Heinz (1922-1944) hervor, der im Krieg fiel. Die ersten sieben Kindheitsjahre verlebte Joachim in Breslau. Bereits vor dem Ende des 2. Weltkrieges musste die Familie ihre Wohnung in Breslau verlassen, weil diese durch Kriegseinwirkungen stark beschädigt worden war. Sie zogen 1944 nach Herrnhut in die Oberlausitz (Sachsen), wo die Mutter zeitweilig ein Kinderheim leitete. Als Joachim zwölf Jahre alt war, verstarb seine Mutter an Krebs. Dieses Ereignis prägte sein späteres Leben nachhaltig, weil er sie lange liebevoll pflegte und damit plötzlich seine wichtigste Bezugsperson verlor.

Von 1943 bis 1950 besuchte Joachim die Grundschule in Breslau und Herrnhut und schloss diese mit der 8. Klasse ab. Schon als Schüler fühlte er sich zu den Insekten hingezogen und interessierte sich für deren Zucht und Präparation. In den schweren Nachkriegsjahren unterstützten ihn seine Eltern wann immer sie konnten, indem sie für Joachim biologische Fachbücher kauften. Durch Lepidopterologen angeregt, führte er schon zwischen 1948 und 1951 erste faunistische Erhebungen in der Umgebung von Herrnhut (Oberlausitz) durch und legte seine erste Insektensammlung (überwiegend Schmetterlinge) an. Bis heute fühlt sich der Jubilar mit der Stadt Herrnhut und der Oberlausitz eng verbunden. Er ist Mitglied in der evangelischen Herrnhuter Brüdergemeine und Ehrenmitglied der Naturforschenden Gesellschaft der Oberlausitz.

\section{Der lange Weg vom biologisch interessierten Schüler zum Wissenschaftler}

An dieser Stelle möchten wir der Übersicht halber voranstellen, dass der weitere Entwicklungsweg von Joachim Oehlke über die Stationen Markkleeberg und Oberholz (1951-1953), Leipzig (1953-1956), Berlin (1956-1961), Potsdam (1961-1962), Berlin-Friedrichshagen (1962-1964) nach Eberswalde (wohnhaft seit 1965) geführt hat. In diese Zeit fällt auch seine erste Ehe (1960-1976) mit Vera Oehlke, geb. Keßler, aus der drei Kinder hervorgingen. Seit 1977 ist Joachim mit der zoologischen Präparatorin Renate Oehlke (geborene Gerlach) verheiratet. Sie brachte zwei Kinder in die zweite Ehe ein, hielt Joachim in allen Lebenslagen den Rücken frei, teilte mit ihm das große Interesse an der Entomologie und sammelte Hymenopteren auf allen gemeinsamen Reisen.

Im Jahre 1951 begann Joachim Oehlke eine zweijährige Berufsausbildung an der Berufsschule für Gartenbau und Landwirtschaft in Markkleeberg, die auch eine berufspraktische Ausbildung auf dem Versuchsgut Oberholz bei Leipzig einschloss. Sowohl die theoretische als auch die praktische Ausbildung zum Landwirtschaftlichen Facharbeiter schloss er mit dem Prädikat „Sehr gut“ ab. Seine Abschlussarbeit fertigte Joachim über tierische Schaderreger (Arthropoden) auf Rinderweiden und im Kulturpflanzenanbau an. Nachdem er am 31. August 1953 seine Lehre beendet hatte, begann er am 1. September des gleichen Jahres eine Ausbildung im biologischlandwirtschaftlichen Studienzweig an der Arbeiter- und Bauern-Fakultät (ABF) der Karl-MarxUniversität in Leipzig. Hier nahm er in den Jahren 1955/1956 auch an der studentischen 
Interessengemeinschaft „Botanik“ teil und legte im Juni 1956 sein Fachabitur mit der Note „Gut“ ab. Zu dieser Zeit vertiefte er auch sein Wissen zur Präparation biologischer Objekte, indem er neben dem Studium im VEB Biologische Lehrmittel Leipzig tätig war. In seiner Freizeit wirkte Joachim damals schon an entomologischen Faunenverzeichnissen für die Umgebung von Leipzig mit (Lepidoptera und Heteroptera) und engagierte sich bei der Erfassung der Wasserkäfer-Fauna in südlichen Teilen der DDR.
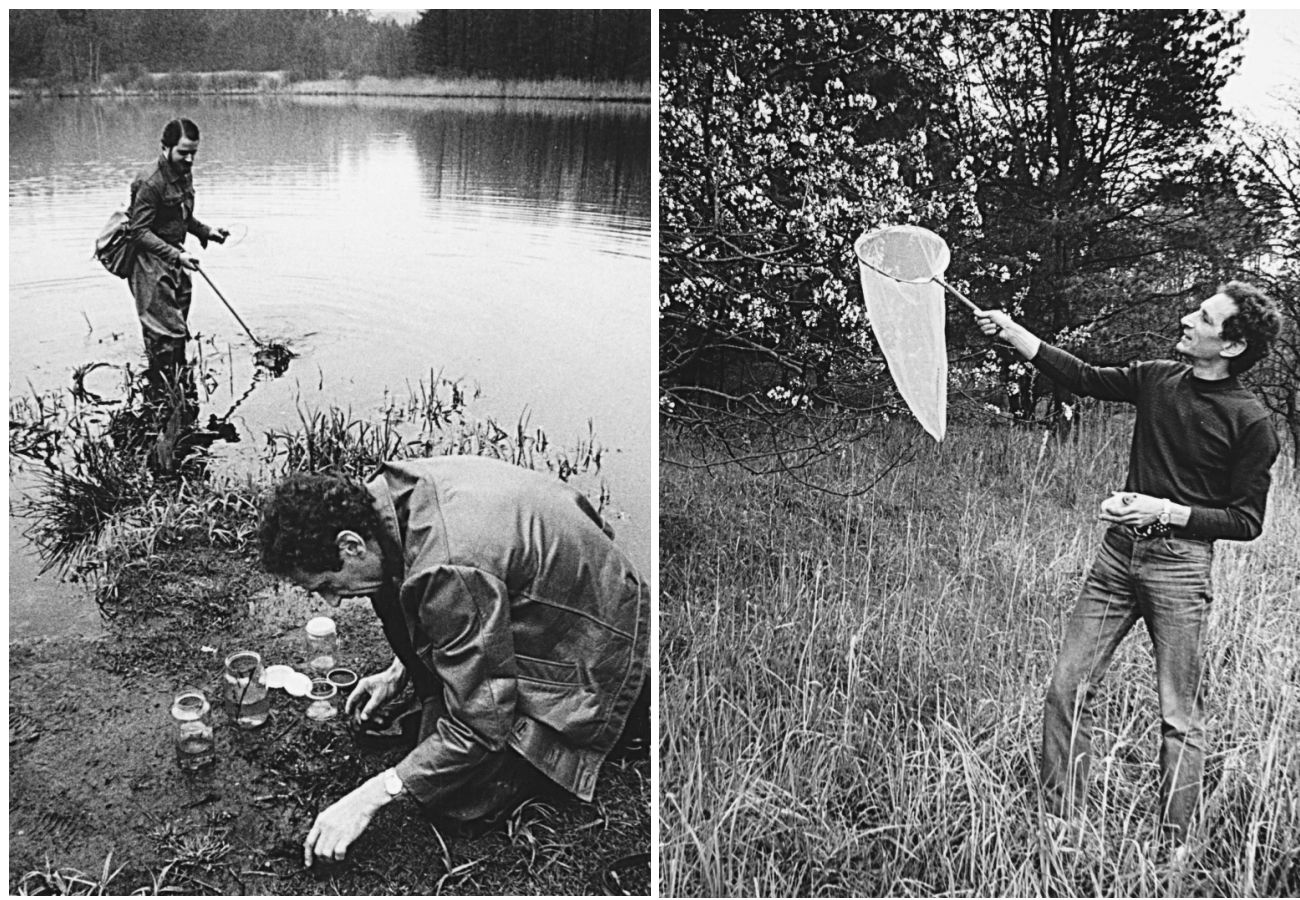

Fig. 2-3: Freilandarbeiten am Eberswalder Stadtsee im März 1983. - 2: Joachim Oehlke (rechts) und Lutz Behne (links) beim Sammeln von Insektenlarven. - 3: Joachim Oehlke beim Kescherfang. Fotos: H. WeHrens.

1956 ging Joachim Oehlke von Leipzig nach Berlin und begann an der MathematischNaturwissenschaftlichen Fakultät der Humboldt-Universität zu Berlin ein Biologiestudium. Dort hörte er unter anderem Vorlesungen bei den Professoren Kästner, Peus, Tembrock, Erdmann, Noack und Nürnberg. Die Diplomhauptprüfung für Biologen bestand er am 13. Juli 1961 mit dem Prädikat „Gut“ und durfte sich fünf Tage später „Diplom-Biologe“ nennen. Damit ging für Joachim ein lange gehegter Wunsch in Erfüllung, denn der direkte Berufsweg zum Biologen blieb ihm wegen der freiberuflichen Tätigkeit seines Vaters verwehrt. Seine Diplomarbeit schrieb er zu dem Thema „Beiträge zur Biologie und Ökologie der Larven einiger Elateridenarten und zur Larvalsystematik der Gattung Agriotes Esch.“.

Während seiner Studienzeit in Berlin war Joachim von 1957 bis 1961 als Hilfsassistent im Institut für Spezielle Zoologie und Zoologischen Museum tätig. Dort führte er Arbeiten in der Hymenopteren-Sammlung aus und wirkte unter Prof. Bischoff und Dr. Steinbach an der Erstellung eines Typenverzeichnisses für parasitoide Hautflügler mit. Die vielseitigen Interessen von Joachim Oehlke kommen auch darin zum Ausdruck, dass er zu dieser Zeit in der helminthologischen Abteilung arbeitete und sich auf die Kratzwürmer (Acanthocephala) - eine kleine Gruppe 
von Darmparasiten mit obligatorischem Wirtswechsel - spezialisierte. Dazu gesellten sich diverse Präparationsarbeiten und Typenermittlungen im Kustodiat Helminthologie (Dr. Hartwig), Praktika über veterinärmedizinisch bedeutsame Arthropoden und Untersuchungen in den Naturschutzgebieten der Insel Hiddensee (Prof. Eichler) sowie die wissenschaftliche Bearbeitung von Kulturpflanzenschädlingen (vor allem Käferlarven) in der Biologischen Zentralanstalt Kleinmachnow (Dr. Noll). Aus dieser breiten Ausbildung und dem Streben, möglichst viel Wissen „aufzusaugen“, erklärt sich auch Joachims besonderes Interesse an der Parasitologie, den Parasitoiden und nicht zuletzt seine besondere Affinität zu den Naturschutzgebieten auf der Insel Hiddensee, die später noch einmal bei der Erforschung der Hymenopteren-Fauna in der DDR (1968-1982) eine größere Rolle gespielt hat.

Im Anschluss an sein Biologiestudium musste Joachim Oehlke zunächst von Berlin nach Potsdam gehen, weil er als Entomologe keine zufriedenstellende Anstellung fand. Von Juli 1961 bis Ende April 1962 war er bei Dr. Schneeweiß im Institut für Getreideverarbeitung Potsdam tätig. Dort arbeitete er zunächst mit Mikroben und Hefen als Backverbesserer für Brot, war Qualitätsprüfer für Backwaren und Tee und beschäftigte sich als Gutachter mit dem Schädlingsbefall in Getreideprodukten. Zudem befasste er sich mit dem Aufbau eines mikrobiologischen Untersuchungslabors und war ab Oktober 1961 als Laborleiter mit speziellen Arbeitsaufgaben in der Entomologie und Mikrobiologie betraut. Während der Zeit in Potsdam blieb Joachim aber auch seinen Hymenopteren treu und bemühte sich um eine dauerhafte Anstellung als Taxonom.

\section{Vom Mitarbeiter zum Leiter des Deutschen Entomologischen Instituts}

Am 2. Mai 1962 war es dann endlich so weit! Joachim Oehlke wurde als wissenschaftlicher Mitarbeiter im Deutschen Entomologischen Institut (DEI) der Deutschen Akademie der Landwirtschaftswissenschaften in Berlin-Friedrichshagen eingestellt. Fortan beschäftigte er sich mit den parasitischen Wespen und übernahm das Kustodiat Hymenoptera. In das gleiche Jahr fällt auch seine erste wissenschaftliche Publikation, ein Beitrag „Zur Larvalsystematik der Gattung Agriotes Esch. (Col. Elateridae)“, in dem er Teilergebnisse aus seiner Diplomarbeit veröffentlichte. In den folgenden Jahren konnte er seine wissenschaftliche Forschungstätigkeit auf die Systematik, Biogeographie und Ökologie der Hautflügler ausrichten, sich mit methodischen Fragestellungen in der Biologie beschäftigen und seine Dissertation in Angriff nehmen. Am 29. September 1965 promovierte Joachim Oehlke an der Mathematisch-Naturwissenschaftlichen Fakultät der Humboldt-Universität zu Berlin zum Doktor der Naturwissenschaften. Die von ihm vorgelegte Dissertation über „Die in europäischen Kiefernbuschhornblattwespen (Diprioninae) parasitierenden Ichneumonidae“ wurde mit dem Prädikat „Sehr gut“ bewertet (Betreuer: Prof. Tembrock).

In der Phase des Umzuges des DEI von Berlin-Fiedrichshagen nach Eberswalde (1964) engagierte sich Joachim zunehmend für den Naturschutz, schrieb Gutachten zur Fauna in Schutzgebieten, interessierte sich für den zoologischen Fließgewässerschutz und übernahm die Leitung der „Arbeitsgemeinschaft Schwärze“. Schon zwei Jahre zuvor hatte er begonnen, zoologische Vorlesungen zu halten und entomologische Praktika für Biolaborantinnen und Präparatoren zu betreuen. Hierbei vermittelte er zoologisches Grundlagenwissen sowie Methoden und Techniken der entomologischen Präparation. In diesem Kontext war Joachim auch als Mitglied von Prüfungsausschüssen bei der Erlangung des Fachschulabschlusses zum Zoologischen Präparator aktiv und engagierte sich in der DDR bei der Erarbeitung von Berufsbildern für den Facharbeiterund Fachschulabschluss zum Zoologischen Präparator. Nach 1989 unterrichtete er zunächst Fernstudenten im Institut für Forstwissenschaften Eberswalde (Umweltbildung \& Forstzoologie), 
übernahm ab 1991 Lehrverpflichtungen an der Fachhochschule Eberswalde (Forstentomologie) und erfüllte Lehraufträge beim Ökologischen Bildungs- und Beschäftigungswerk Brandenburg sowie bei der Zukunftswerkstatt Arbeit und Bildung in Peetzig (Zoologie \& Naturschutz).

In der Zeit des politischen Umbruchs in der DDR und der Wiedervereinigung Deutschlands wurde der parteilose Joachim Oehlke von den wissenschaftlichen Mitarbeitern zum Direktor des Deutschen Entomologischen Instituts gewählt. Niemand von uns wusste damals, wohin die „Reise“ geht und wie das Institut mit der großen Forschungssammlung und der Entomologischen Bibliothek nach der Währungsunion weiter am Leben zu halten war. Am 1. Oktober 1990 übernahm Joachim die Dienstgeschäfte von Prof. Dr. Werner Ebert, der vor seinem Ruhestand von Juli bis September 1990 als geschäftsführender Direktor fungierte (Rohlfien \& Kroel 1995: 19). Von diesem Tag an ging er bei den Bundes- und Landesministerien „Klinken putzen“ und überführte das Institut von der Biologische Zentralanstalt Berlin (1990-1991) über diverse Förderungen im Wissenschaftler-Integrations-Programm des Erneuerungsprogramms für Hochschule und Forschung in den neuen Bundesländern (1992-1993) als „Projektgruppe Entomologie“ in die Fachhochschule Eberswalde (1993-1996).
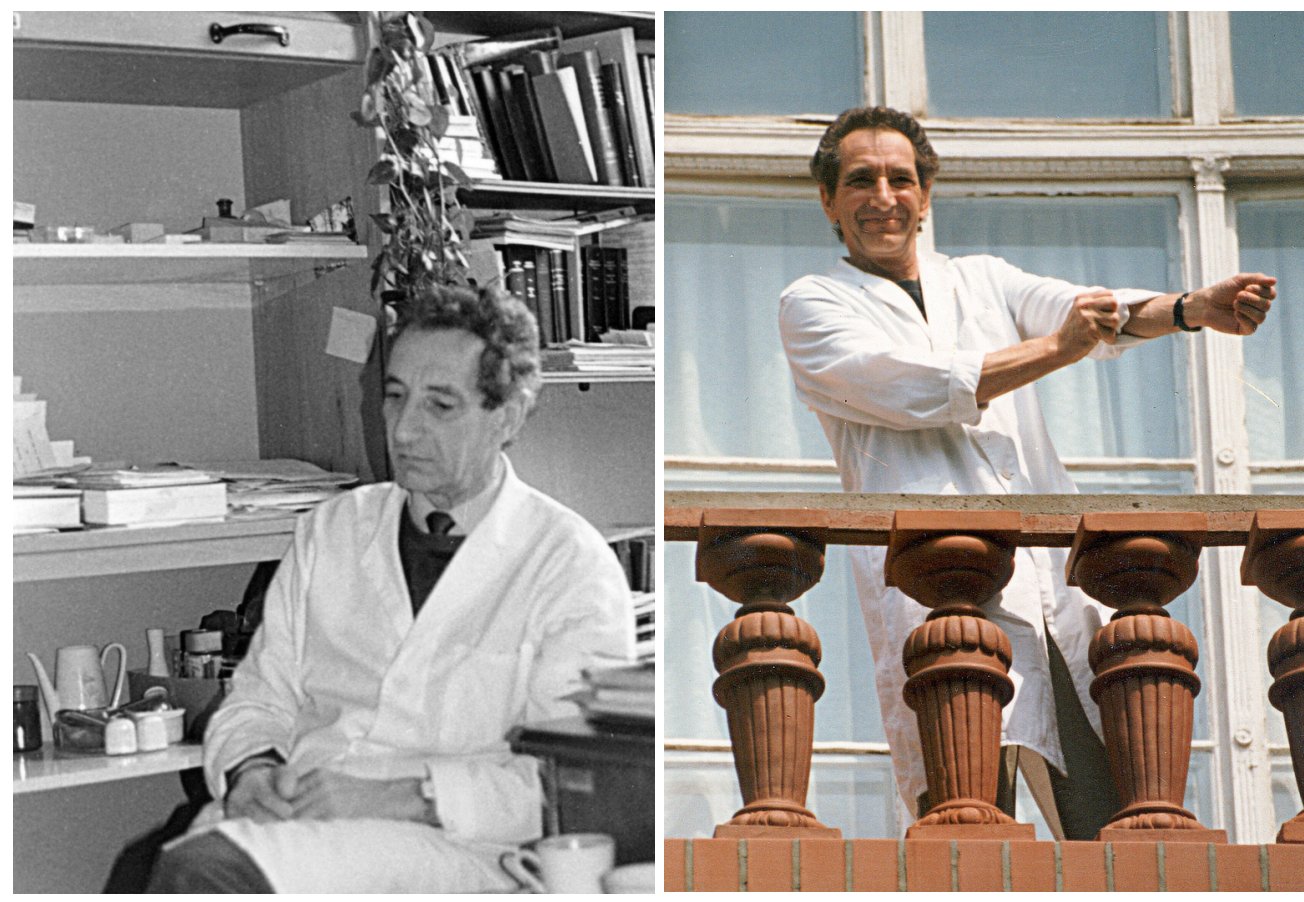

Fig. 4-5: Joachim Oehlke als Leiter des Deutschen Entomologischen Instituts in Eberswalde. - 4: Beim Pressegespräch in seinem Arbeitszimmer (Januar 1991). - 5: "Ärmel hoch und angepackt!« Joachim nach dem Abschluss denkmalpflegerischer Arbeiten auf dem Balkon des Institutsgebäudes (Sommer 1993). Fotos: R. \& A. Richter und H. Wehrens (Archiv des SDEI).

Trotz widriger Rahmenbedingungen gelang es Joachim Oehlke, die Arbeitsfähigkeit des DEI mit allen Mitarbeitern in der Bibliothek und der Forschung aufrecht zu erhalten. Durch seinen beharrlichen, unermüdlichen Einsatz konnten in dieser schweren Zeit immer wieder Projektgelder eingeworben werden, die den Erhalt und den Fortbestand des Hauses als eine in sich geschlossene Forschungseinrichtung sicherstellten. Sein Verdienst ist es auch, dass wichtige Sanierungsarbeiten 
am Dach, der Fassade und den Fenstern des Eberswalder Institutsgebäudes durchgeführt wurden. Dadurch konnten die wertvollen Insektensammlungen und Bücher besser geschützt werden.

Einige Monate vor seinem Ausscheiden aus dem DEI wurde am 23. September 1993 der „Verein der Freunde und Förderer des Deutschen Entomologischen Instituts e.V.“ gegründet, dessen Vorsitzender er von 1993 bis 2003 war und dem er noch heute angehört.

Tab. 1: Insektenarten, die von Joachim Oehlke beschrieben wurden.

\begin{tabular}{|c|c|c|c|}
\hline Ordnung & Familie & Art & Literaturquelle \\
\hline Hymenoptera & Crabronidae & $\begin{array}{l}\text { Psen (Mimesa) tenuis } \\
\text { OEHLKe, } 1965\end{array}$ & OEHLKe, 1965: 343 \\
\hline Hymenoptera & Diapriidae & $\begin{array}{l}\text { Cinetus balticus } \\
\text { SzABó \& OEHLKE, 1986: } 105\end{array}$ & SZABó \& OEhlKe (1986): 105 \\
\hline Hymenoptera & Ichneumonidae & $\begin{array}{l}\text { Neoxorides montanus } \\
\text { OEHLKE, } 1966\end{array}$ & OEHLKe (1966): 889 \\
\hline Hymenoptera & Megaspilidae & $\begin{array}{l}\text { Lagynodes pseudocarinatus } \\
\text { SzABó \& OEHLKE, } 1986\end{array}$ & Szabó \& OEhlKe (1986): 99 \\
\hline Hymenoptera & Proctotrupidae & $\begin{array}{l}\text { Cryptoserphus koggeauxilliarius } \\
\text { SzABó \& OEHLKE, } 1986\end{array}$ & Szabó \& OEhlKe (1986): 100 \\
\hline Hymenoptera & Scelionidae & $\begin{array}{l}\text { Idris ilonkae } \\
\text { SzABó \& OEHLKE, } 1986\end{array}$ & Szabó \& OEHLKe (1986): 105 \\
\hline Hymenoptera & Scelionidae & $\begin{array}{l}\text { Macroteleia renatae } \\
\text { SzABó \& OEHLKE, } 1986\end{array}$ & Szabó \& OEhlKe (1986): 100 \\
\hline
\end{tabular}

Tab. 2: Insektenarten, die nach Joachim Oehlke benannt wurden.

\begin{tabular}{|l|l|l|l|}
\hline Ordnung & Familie & Art & Literaturquelle \\
\hline Coleoptera & Staphylinidae & $\begin{array}{l}\text { Coryphiodes oehlkei } \\
\text { ZerCHE, 1990 }\end{array}$ & ZERCHE (1990): 78 \\
\hline Hymenoptera & Braconidae & $\begin{array}{l}\text { Orgilus oehlkei } \\
\text { TAEGER, 1989 }\end{array}$ & TAEGER (1989): 144 \\
\hline Hymenoptera & Braconidae & $\begin{array}{l}\text { Apanteles oehlkei } \\
\text { PAPP, 1982 }\end{array}$ & DATHE (2010): 55 \\
\hline Hymenoptera & Colletidae & $\begin{array}{l}\text { Hylaeus (Hylaeus) oehlkei } \\
\text { DATHE, 2010 }\end{array}$ & MoMOI (1973): 231 \\
\hline Hymenoptera & Ichneumonidae & $\begin{array}{l}\text { Coccygomimus oehlkei } \\
\text { MomOI, 1973 }\end{array}$ & HeINRICH (1972): 95 \\
\hline Hymenoptera & Ichneumonidae & $\begin{array}{l}\text { Platylabus oehlkei } \\
\text { HeINRICH, 1972 }\end{array}$ \\
\hline Hymenoptera & Tenthredinidae & $\begin{array}{l}\text { Pachynematus oehlkei } \\
\text { Muche, 1973 }\end{array}$ & MuCHE (1973): 167 \\
\hline Hymenoptera & Vespidae & $\begin{array}{l}\text { Stenodynerus oehlkei } \\
\text { GusENLEITNER, 2008 }\end{array}$ &
\end{tabular}




\section{Joachim Oehlke als Hymenopterologe}

Die wissenschaftliche Tätigkeit von Joachim Oehlke am Deutschen Entomologischen Institut war auf die Taxonomie und Diversität der Hautflügler ausgerichtet. Von ihm sind lediglich 7 Hymenopteren-Arten beschrieben worden (Tab. 1) - ein Hinweis darauf, dass Joachim schon früh seine Berufung eher darin sah, Informationen zur Biologie und Verbreitung paläarktischer Spezies zusammen zu tragen, nomenklatorische Probleme aufzuklären und die Arten mit figurierten Bestimmungstabellen erkennbar zu machen. Diese Leistungen honorierten in- und ausländische Wissenschaftler mit Dedikationen, so dass 8 Insektenarten heute seinen Namen tragen (Tab. 2).

Joachim Oehlke hatte einen besonderen Einfluss auf die Erforschung der Hautflügler, weil er Bestimmungsschlüssel verfasste, die anderen den Zugang zu dieser Gruppe erleichterten. In einer Zeit, wo noch niemand an Computer und Internet dachte, waren für ihn die Verfügbarkeit von Literatur und Sammlungsdaten von grundlegender Bedeutung. Damit unterstützte er auch andere Hymenopterologen aktiv bei deren Forschung. So wurde zum Beispiel ein Auto mit historischer Literatur aus der Institutsbibliothek beladen und zu W. H. Muche nach Radeberg gefahren, um ihn bei der Klärung von Fragen zur Nomenklatur von Pflanzenwespen zu unterstützen. Im Rahmen der entomologischen Fachgruppen des Kulturbundes suchte er persönlichen Kontakt zu anderen Hymenopterologen und gestaltete die jährlich stattfindenden Spezialistenlager im Bezirk Frankfurt (Oder) maßgeblich mit. Seine Einstellung zur Arbeit von „Amateuren“ wird klar, wenn man weiß, dass er diesen Begriff ablehnt und stattdessen stets von „Freizeitforschern“ spricht. Seine Mitarbeit an der „Insekten-Fauna der DDR“ ist wahrscheinlich der wirksamste Beitrag für die Einbeziehung von Freizeitforschern in die Bearbeitung der Hymenopteren.

Joachims erste große Publikation war seine Promotionsarbeit über die in Kiefernbuschhornblattwespen parasitierenden Schlupfwespen (1965). Es folgten der Bestimmungsschlüssel für die Unterfamilien der Hymenoptera (1969) und die Spheciden-Fauna der DDR (1970), die einen wesentlichen Anteil an der Verbesserung der Determinationsmöglichkeiten in der Gruppe hatten. Die in den Folgejahren erhöhten Aktivitäten in der Grabwespenforschung (seit 1972) sind ebenso offensichtlich, wie sein Interesse bei der Erarbeitung von Bestimmungswerken (1978-2011) und Roten Listen (1992, 1998). An dieser Stelle müssen vor allem seine Beiträge zu den Hautflüglern (Hymenoptera) und Fächerflüglern (Stresiptera) in dem weithin bekannten "Stresemann“, der Exkursionsfauna für Deutschland (4.-11. Auflage) hervorgehoben werden. Sie haben zahlreichen entomologisch Interessierten, Studenten und Freizeitforschern einen Einstieg in diese Insektengruppen ermöglicht. Neben seinen taxonomisch-faunistischen Arbeiten und größeren Übersichtswerken arbeitete Joachim auch immer an der Klärung der Nomenklatur der Hymenopteren. Die Bedeutung von Katalogen hat er stets klar vertreten, und mit seinem Katalog der westpaläarktischen Ephialtinae (1967) auch praktisch umgesetzt. Weniger bekannt ist außerhalb des DEI, dass dieser Katalog von ihm und seinen Mitarbeiterinnen über viele Jahre für alle westpaläarktischen Ichneumonidae auf Karteikarten weitergeführt wurde und eine Publikation der Ergebnisse vorbereitet war. Mit der politischen Wende 1989 änderten sich die personellen Voraussetzungen für diese Arbeit und Joachims Prioritäten, indem er im Überlebenskampf des Institutes die Zügel in die Hand nahm und das DEI vor der „Abwicklung“ bewahrte. Mit dem Erscheinen des ersten elektronischen Schlupfwespenkataloges von D. Yu Anfang der 90er Jahre erübrigte sich schließlich die Publikation des Westpaläarktis-Kataloges. 


\section{Verdienste um die Insektenausstellung in Eberswalde}

Nachdem Joachim Oehlke im Oktober 1990 die Leitung des DEI übernommen hatte, kam seine hymenopterologische Forschung weitgehend zum Erliegen und administrative Dinge traten in den Vordergrund. In zunehmendem Maße waren tragfähige Forschungskonzepte zu erarbeiten, mussten neue Kooperationspartner gefunden und potentielle Geldgeber umworben werden, die zum Fortbestand des Institutes beitragen konnten. Sehr schnell wurde klar, dass das nur zu erreichen war, wenn man eine intensive Lobbyarbeit betrieb, Projektgelder einwarb und die breite Öffentlichkeit für die Insektenforschung am DEI sensibilisierte. Folglich erkannte Joachim schon sehr früh, dass das Institut mit seiner wissenschaftlichen Expertise und der einzigartigen entomologischen Fachbibliothek nicht nur in der gesamtdeutschen Wissenschaftslandschaft neu auszurichten bzw. zu verankern war, sondern auch in der breiten Öffentlichkeit dauerhaft wahrgenommen und in den Printmedien präsent sein musste.
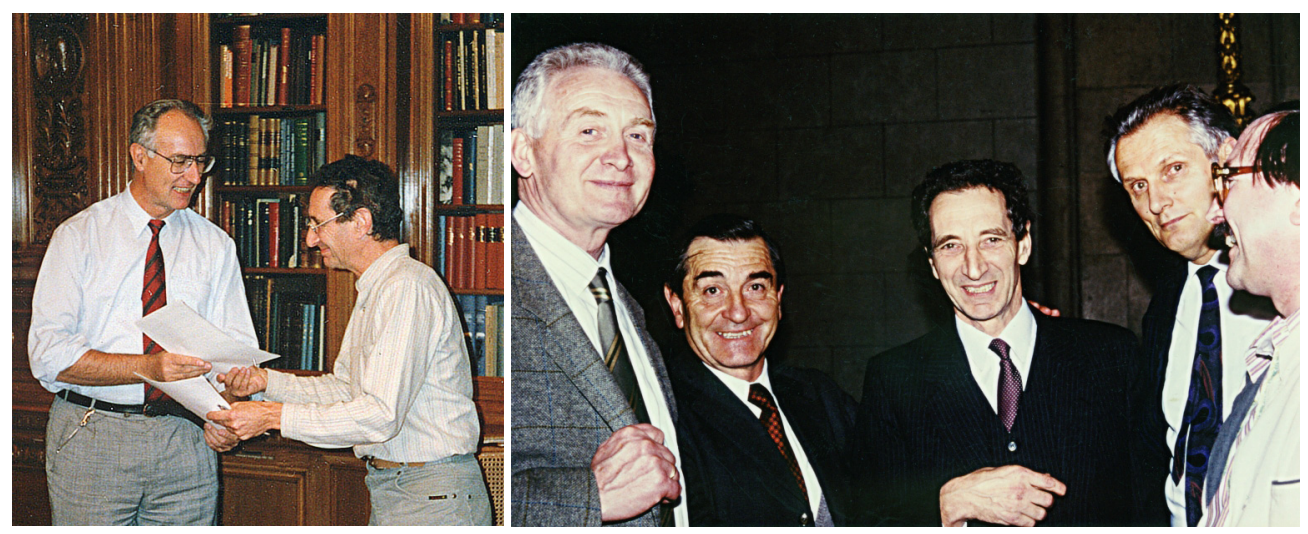

Fig. 6-7: Joachim Oehlke als anerkannter Wissenschaftler und Verhandlungspartner. - 6: Zusammen mit Prof. Dr. C. M. Naumann (links) beim Abschluss des Partnerschaftsvertrages zwischen dem Deutschen Entomologischen Institut und dem Forschungsinstitut und Museum Alexander Koenig am 28. November 1990 in Bonn. - 7: Im Gespräch mit Teilnehmern der Entomologen-Tagung von ÖEG, AGÖE, SEG und DGaaE, 2.-6. April 1991 in Wien. Fotos: DEI und V. Cıоchia (Archiv des SDEI).

Aus diesen Gründen wurde im September 1992 die populärwissenschaftliche Ausstellung „Insekten - Faszinationen in Natur, Sammlung und Buchkunst“ etabliert, die einen kleinen Einblick in die formen- und farbenprächtige Welt der Insekten bot. Mit der Exposition betrat das DEI völliges Neuland. Wenngleich das Institut einst als „Deutsches Entomologisches National-Museum " gegründet wurde, hatte es - anders als der Gründungsname vermuten lässt - vorher zu keiner Zeit eine museale Ausstellung gegeben. Geschickt verstand Joachim Oehlke es, zusätzliche Mittel für die „Präsentation der Forschungsergebnisse“ aufzutreiben, denn für den Aufbau einer öffentlich zugänglichen Ausstellung sah der Etat keine Gelder vor. Als außerordentlich verdienstvoll ist in diesem Zusammenhang hervorzuheben, dass es ihm sogar gelang, zusätzliches Personal für die Ausstellungsbetreuung einzustellen. Dabei handelte es sich um drei Mitarbeiterinnen, die so für neun Jahre in Lohn und Brot standen. Die Ausstellung mit Präparaten, Insektenliteratur und Insektarien bescherte dem Institut jährlich bis zu 4500 Besucher. Welche Popularität die Ausstellung erfuhr, ist exemplarisch vielleicht besonders eindrucksvoll an der Reaktion der Öffentlichkeit abzulesen, als sie im Zuge der Institutsanbindung an das Leibniz-Zentrum für Agrarlandschaftsforschung (ZALF) schließen musste. So berichtete die Presse mehrfach fälschlich, dass das ganze Institut nun geschlossen 
worden sei; ein Zeichen dafür, dass die Ausstellung - quasi als „viertes Standbein“ neben der Forschung, der wissenschaftlichen Sammlung und der Bibliothek - etabliert war und von der Öffentlichkeit in Brandenburg als bildungspolitisches Kleinod wahrgenommen wurde. Ihr Wegfall im Oktober 2001 ist und bleibt ein schmerzlicher Verlust, denn neben zahlreichen Exponaten rund um das Thema „Insecta“ wurden in der größten Insektenexposition Ostdeutschlands bis zu 56 verschiedene Arten lebend gezeigt (BEDERKe 2001). Bis heute gibt es Anrufer oder sogar Besucher, die plötzlich in Müncheberg vor der Tür stehen und nach der Ausstellung fragen.

\section{Die entomologischen Sammlungen von Joachim Oehlke}

Als Insekten-Sammler hat Joachim Oehlke umfangreiche Bestände für das Deutsche Entomologische Institut zusammengetragen. Sein Arbeitsschwerpunkt waren die Ichneumonidae, Sphecidae, Vespidae und Pompilidae. Aber auch andere Insektengruppen ignorierte er auf seinen Sammelreisen nicht. Die meisten Hymenopteren, die Joachim vor der Wende sammelte, stammen aus DDR. Aber auch Ausbeuten von seinen zahlreichen Auslandsreisen (vor allem nach Südosteuropa und in die mittelasiatischen Gebiete der früheren Sowjetunion) sind zu großen Teilen im DEI deponiert. Auch nach 1990 gelangten noch kleinere Aufsammlungen (diverse Insecta aus tropischen und subtropischen Gebieten Afrikas, Asiens, Mittel- und Südamerikas; excl. Hymenoptera) nach Eberswalde bzw. Müncheberg (Autorenkollektiv 1993-2010). Über 11000 genadelte Exemplare (excl. Hymenoptera) - darunter Ameisen, Heuschrecken, Käfer und Schmetterlinge - wurden im Januar 2012 per Schenkung an das Naturkundemuseum Potsdam übergeben (DitTfELd 2012). Seine Inklusen-Sammlung wird voraussichtlich im Dezember 2012 an die Familie Hofferns (Hamburg) veräußert. Sie besteht aus ca. 200 Stücken Baltischem Bernstein mit pflanzlichen und tierischen Einschlüssen (Insekten aller Ordnungen) und enthält 6 Holotypen (Hoffeins \& Hoffeins, pers. Mitt.). Die umfangreiche Hautflügler-Sammlung (Hymenoptera) wird dagegen noch am Wohnsitz der Familie Oehlke in 16225 Eberswalde, Schillerstraße 2 aufbewahrt.

\section{Joachims »außerberufliche« Aktivitäten}

Bis heute ist Joachim ein kritischer, streitbarer Wissenschaftler, der vielseitige Interessen besitzt und sich neben seinem Beruf als Entomologe immer gesellschaftlich positioniert hat. In den frühen Jahren war er zum Beispiel als Sozialhelfer in Berlin-Prenzlauer Berg tätig, engagierte sich in der Jugendarbeit des Kulturbundes der DDR und leistete in viel beachteten Projekten eine enorme Naturschutzarbeit.

Neben seinem Hauptberuf als Entomologe und Hochschullehrer war Joachim Oehlke in mehreren Gesellschaften und Vereinen tätig und übte in ihnen diverse Funktionen aus (siehe nachfolgende Kapitel). Über den „Verein der Freunde und Förderer des Deutschen Entomologischen Instituts“ und die „Naturforschende Gesellschaft der Oberlausitz“ wurde schon an anderer Stelle kurz berichtet. Außerdem ist er ein aktives Mitglied der DGaaE, der EFG, des NMVB, von Greenpeace, des NABU, der ZAG Wirbellose und des Rotary Clubs Eberswalde-Barnim. Bei der Nennung der vorstehenden Vereinigungen fällt auf, dass er bis heute ein feuriger Verfechter des Natur- und Umweltschutzes ist. Dabei waren bzw. sind ihm die „Arbeitsgemeinschaft Schwärze“ (1965-1995), die "Gesellschaft für Natur und Umwelt“ im Kulturbund der DDR (1980-1992) und die „Zentrale Arbeitsgemeinschaft Wirbellose im Terrarium“ (seit 1999) besonders ans Herz gewachsen. Nach der Wiedervereinigung erwarb sich Joachim zudem 
große Verdienste bei der nahtlosen Überleitung der im Kulturbund der DDR vereinten Naturschützer in den Naturschutzbund Deutschland (NABU). Als Leiter des deutschlandweit einzigen Instituts, das sich ausschließlich der Insektenkunde verschrieben hat, nahm Joachim Oehlke seine Verantwortung in den entomologischen Fachorganen des Kulturbundes und später des NABU wahr. So bekleidete er von 1986 bis 1993 als Leiter des Zentralen Arbeitskreises Hymenoptera, als Mitglied des Landesfachausschusses Entomologie und als erster stellvertretender Vorsitzender im Bundesfachausschuss Entomologie Schlüsselpositionen. Mit seinen weit reichenden Kontakten vernetzte er die Aktivitäten der „Umweltaktivisten“, sorgte dafür, dass der NABU-Kreisverband Barnim räumlich eine Heimat fand und stattete ihn mit einer Geschäftsführerstelle aus. Die Finanzierung dafür musste gut begründet werden und vor dem Erfolg lag ein Wust an bürokratischen Hürden. Aber Joachim hat sich Verbündete gesucht und die Sache mit Beharrlichkeit durchgekämpft, auch wenn der tägliche Kleinkram von den wirklich wichtigen Problemen ablenkte. Diesem schon mehrfach geschilderten Durchhaltewillen, der aus seiner ehrlichen Überzeugung resultiert, verdanken wir heute unter anderem die Sicherung des Truppenübungsplatzes Trampe als FFH-Gebiet und den Erwerb des Pimpinellenberges durch den NABU-Landesverband Brandenburg. Sein fachlicher Rat war zudem über Jahrzehnte in vielen gesellschaftlichen Gremien und staatlichen Verwaltungen gefragt. So wirke er von 1995 bis 2004 zunächst als Mitglied und später als Vorsitzender des Naturschutzbeirates bei der unteren Naturschutzbehörde des Landkreises Barnim mit.

Eine ganz andere Facette in seinem Leben ist der Sport. Dabei hat sich Joachim ganz den Kampfsportarten verschrieben. Lange Zeit war er ein sehr aktiver Judosportler, der den Schwarzen Gürtel trägt und den 2. Dan ablegte. Der 3. Dan wurde ihm in Anerkennung seiner langjährigen, engagierten Nachwuchsarbeit verliehen. Zudem war er lange Zeit Übungsleiter und betätigte sich von 1967 bis 1975 als Judo-Kampfrichter im Bezirk Frankfurt (Oder). Auch hier scheute er sich nicht vor der Verantwortung und war jahrelang Vorstandsmitglied im Sportverein Dynamo Eberswalde, Abteilung Judo (später Polizeisportverein Eberswalde). Auch heute geht er noch regelmäßig zum Sport und beginnt den Tag nicht ohne die obligatorischen Liegestütze.

Dagegen setzte Joachim sein enormes Fachwissen über die Insektenpräparation, die er sich seit den 1950er Jahren angeeignet hatte, nebenberuflich auch bei der Herstellung von biologischen Lehrmitteln ein. Zusammen mit seiner Frau Renate produzierte und vertrieb er über 35(!) Jahre (von 1972 bis 2007) entomologische Anschauungsobjekte für Schulen, Hochschulen, Museen, forstliche Einrichtungen, den Pflanzenschutz und die Schädlingsbekämpfung. Als alleinige Hersteller in der DDR konnten sie jedoch den enormen Bedarf an Anschauungskästen (vor allem von Haus-, Vorrats- und Materialschädlingen, ihren Larvenformen sowie den charakteristischen Schadbildern) nicht abdecken. Nur wenige wissen, dass die daraus resultierenden Einnahmen zu einem beträchtlichen Teil in den Ankauf von Insektenkästen, den Erwerb von kleineren entomologischen Sammlungen und in die zahlreichen Sammelreisen von Renate und Joachim investiert wurden. Eine lohnende Geldanlage, wenn man sich heute ihre Hymenopteren-Kollektion in Eberswalde anschaut.

\section{Joachim Oehlke als Hochschullehrer}

Am 1. Dezember 1993 wurde Joachim als Professor für „Angewandte Tierökologie“ an die Fachhochschule Eberswalde (heute Hochschule für nachhaltige Entwicklung), Fachbereich Landschaftsnutzung und Naturschutz, berufen. Damit war zugleich sein Ausscheiden aus dem Deutschen Entomologischen Institut verbunden, das mit diesem formalen Akt als „Projektgruppe Entomologie" in die Fachhochschule Eberswalde integriert werden konnte. Am gleichen Tag 
übergab Joachim Oehlke die Leitung und Dienstgeschäfte des DEI an Prof. Dr. sc. Holger H. Dathe (Autorenkollektiv 1993: 5). Dieser Anbindung an die Fachhochschule verdankten damals 33 Mitarbeiterinnen und Mitarbeiter, dass sie weiterhin eine berufliche Perspektive hatten. Für Joachim Oehlke hingegen bedeutete dieser Schritt, dass er sich von der hauptberuflich ausgeübten Hymenopterologie verabschieden musste. Seitdem bestimmten vor allem der Aufbau des Lehrstuhls und die studentische Ausbildung seinen Arbeitsalltag. In seine Zeit als Hochschullehrer fällt auch die Erarbeitung eines aufwendig und breit angelegten Leit- und Zielartenkataloges wirbelloser Tiere für das Bundesland Brandenburg (1996). Dieser wurde von ihm im Rahmen eines Forschungsprojektes, das er unter Einbeziehung zahlreicher Experten konzipierte und koordinierte, maßgeblich mit erarbeitet.

Bei der inhaltlichen Ausgestaltung der Lehre wie auch beim Aufbau einer Material- und Lehrsammlung hat Joachim Oehlke Pionierarbeit geleistet. Die Lehre richtete er von Beginn an auf Tierökologie und zoologische Systematik aus, weil ihm klar war, dass es eine praxisbezogene „Angewandte Tierökologie“ ohne Formenkenntnis und systematisches Wissen nicht geben kann. Er schöpfte dabei aus seinem breiten Fundus an entomologischem Fachwissen, konnte darüber hinaus jedoch auch Kenntnisse aus seiner langjährigen, ehrenamtlichen Praxisarbeit (z. B. in der Fisch- und Gewässerökologie) einfließen lassen. Zugleich profitierte er von seinen umfangreichen Reiseerfahrungen, die er auf fast allen Kontinenten und in den verschiedensten Klimazonen sammelte.

Joachim beherrschte die ganze Palette zoologisch-ökologischer Lehre, bestehend aus Vorlesungen, Übungen und Geländepraktika. Unter letzteren waren seine kombinierten Lichtfang-Grillabende, die er mit seiner Ehefrau Renate im Gartenhaus in Melchow ausgerichtete, geradezu legendär. Er begeisterte interessierte Studenten für (auch körperlich) anspruchsvolle, naturschutzbezogene Freilandforschung. Bei seiner Lehrtätigkeit kam ihm auch immer seine kontinuierliche Verankerung in der Naturschutzpraxis und in der Naturschutzpolitik zugute. Joachim hat seine exponierte Stellung (ob als Leiter des DEI oder als Hochschullehrer) zudem stets als Aufgabe verstanden, sich über den Beruf hinaus für konkrete naturschutzfachliche Themen und naturschutzpolitische Ziele einzusetzen. Dabei scheute er auch vor Auseinandersetzungen mit Vertretern von Behörden nicht zurück, wenn es der Sache dienlich war. Wer den streitbaren Joachim Oehlke kennt, weiß, dass konfliktreiche Situationen zu seinem Leben gehören wie das Salz zum Brot. Sein leidenschaftlicher, uneigennütziger und oft energischer Einsatz haben ihm in seiner Laufbahn als Biologe, Naturschützer und Leiter in beiden Gesellschaftssystemen nicht immer zum Vorteil gereicht. Sie brachten ihm aber Achtung und Anerkennung bei seinen Mitstreitern, Studenten und unterstellten Mitarbeitern ein. Ein Pfand, mit dem er oft aussichtslos scheinende Dinge anging und aus dem er die Kraft schöpfte, anstehende Probleme beharrlich und zielgerichtet zu lösen.

Joachims Lehrtätigkeit an der Fachhochschule Eberswalde endete mit der Emeritierung am 31. März 2001. Anlässlich seines Ausscheidens aus dem aktiven Berufsleben fand am 6. April 2001 ein Ehrenkolloquium in der Aula der Fachhochschule statt, an dem viele Mitarbeiter und Studenten, zahlreiche Gäste und langjährige Wegbegleiter aus dem ganzen Bundesgebiet teilnahmen. Auf der Festveranstaltung wiesen die Gastredner nicht nur auf Joachims Leistungen als Wissenschaftler und Hochschullehrer gebührend hin, sondern hoben auch seinen langjährigen, engagierten Einsatz für den Naturschutz hervor, der an diesem Tag mit der Ehrennadel des NABU in Gold gewürdigt wurde (ReDAKTION MOZ 2001).

Nach seiner Verabschiedung aus dem Hochschuldienst (Loose 2001), der Übergabe seiner biologischen Lehrmittelfirma an einen engagierten Nachfolger (REDAKTION MOZ 2007) und seinem schmerzlichen Rückzug aus fast allen leitenden Gremien (u. a. Redaktion MOZ 2009) widmet 
sich Joachim wieder mehr seinem Steckenpferd, dem Sammeln, Präparieren und Bestimmen von Hymenopteren (Vespidae). Dabei trifft der Leitspruch „Rentner haben niemals Zeit“ in besonderer Weise auf den nicht ruhenden Jubilar zu, der heute (oft mehrmals im Jahr) in ferne Länder reist, seine Sammelausbeuten an Experten in der ganzen Welt verschickt und so seine Hymenopteren-Kollektion unermüdlich mit wertvollem, solide bestimmtem Material vervollständigt. Wir wünschen Joachim gute Gesundheit, viel Freude im Kreise seiner Familie und weitere erfolgreiche Jahre in seiner wissenschaftlichen Tätigkeit.

\section{Sammelreisen und Expeditionen außerhalb Deutschlands}

Von Joachim Oehlke wurden bisher 52 Länder bereist, in denen er (oft zusammen mit seiner Frau Renate) neben anderen Insektenarten vor allem Hautflügler (Hymenoptera) gesammelt hat. Für die bereisten Staaten wurden die gegenwärtig gültigen Länderbezeichnungen verwendet. Das trifft vor allem auf die Tschechoslowakei und die Republiken der früheren Sowjetunion zu.

AFROTROPIS: Guinea (2003); Kamerun (2006); Madagaskar (1997, 2003); Namibia (2010); Malawi (2008); Südafrika (1999, 2012); Tansania (2008); Togo (2008); Uganda (2012).

NEARKTIS: Vereinigte Staaten von Amerika (1989).

NEOTROPIS: Chile (2007); Costa Rica (1998); Dominikanische Republik (2005); FranzösischGuyana (2009); Jamaika (2005); Kuba (2001, 2007, 2009, 2012); Mexiko (2000); Nikaragua (1993); Peru (2004); Venezuela (1998).

ORIENTALIS: Indonesien (1994); Laos (1996); Malaysia (2011); Thailand (1996, 2002).

PALÄARKTIS: Armenien (1997); Aserbaidschan (1997); Bulgarien (1967, 1979, 1985); China (1992), Georgien (1987, 1984, 1990); Griechenland (2012); Israel (1993, 1994); Kasachstan (1983, 1989); Kirgisien (1983, 1984, 1987, 2007); Kroatien (2010); Lettland (1998, 2002); Mongolei (1988); Niederlande (1992); Österreich (2006, 2008, 2009, 2011); Polen (1971, 1977, 1978, 1980, 2011); Portugal (1996 Madeira, 2000 Algarve); Rumänien (1985); Russland (1968, 1983, 1984 Moskauer Gebiet; 1984 Kaukasus); Slowakei (1972, 1974, 1978, 1986, 1989);
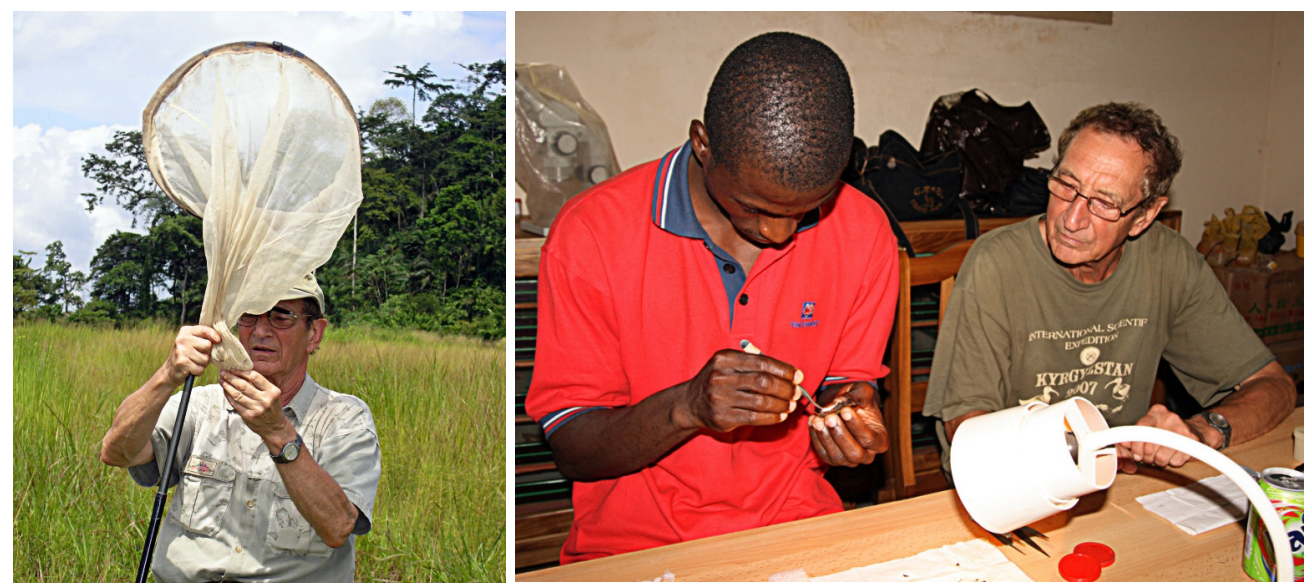

Fig. 8-9: Joachim Oehlke als Expeditionsteilnehmer in Afrika. - 8: Beim Hymenopteren-Fang im Südwesten Kameruns (Mai 2006). - 9: Beim Unterweisen von Studenten in entomologische Präparationstechniken an der Université de Lomé in Togo (April 2008). Fotos: F. Menzel. 
Spanien (1990 Costa Brava, 1995 Kanarische Inseln); Sudan (1996); Tadschikistan (1989); Tschechien (1964, 1966); Tunesien (1991); Turkmenien (1987, 1989); Ungarn (1966, 1980, 1982, 1983, 1985, 1990, 1992, 1995, 1997, 1999, 2003); Usbekistan (1984, 1987, 1989); Weißrussland (1984); Zypern (2008).

\section{Mitgliedschaft in Vereinen und Gesellschaften}

- Biologische Gesellschaft der Deutschen Demokratischen Republik, Berlin (1984-1990)

- Brandenburgischer Kulturbund e.V., Potsdam (1990-1991

- Deutsche Gesellschaft für allgemeine und angewandte Entomologie e.V. (DGaaE), Giessen (seit 1991)

- Entomofaunistische Gesellschaft e.V. (EFG), Dresden (seit 2005)

- Gesellschaft für Natur und Umwelt (GNU) im Kulturbund der DDR, Berlin (1980-1990)

- Greenpeace e.V., Hamburg (seit 1993)

- International Society of Hymenopterists (ISH), Ottawa (1992-1997)

- Naturforschende Gesellschaft der Oberlausitz e.V., Görlitz (seit 1995)

- Naturkundlicher Museumsverein Brandenburg e.V. (NMVB), Potsdam (seit 2003)

- Naturschutzbund Deutschland e.V. (NABU), Bonn/Berlin (seit 1992)

- PHYLLODROM e.V., Museum und Institut für Regenwaldökologie, Leipzig (1994-2003)

- Rotary International, Rotary Club Eberswalde-Barnim, Eberswalde (seit 1993)

- Verein der Freunde und Förderer des Deutschen Entomologischen Instituts e.V., Eberswalde/ Müncheberg (seit 1993)

- World Wide Fund For Nature Deutschland (WWF), Frankfurt am Main (1990-2006)

- Zentrale Arbeitsgemeinschaft Wirbellose im Terrarium e.V. (ZAG Wirbellose), Chemnitz/ Wernigerode (seit 1999)

\section{Ausgeübte Funktionen}

- 1968-1973: Mitglied der Zentralen Prüfungskommission für zoologische Präparatoren beim Ministerium für Kultur der DDR

- 1980-1990: Mitglied des Kreisvorstandes Eberswalde der Gesellschaft für Natur und Umwelt beim Kulturbund des DDR

- 1984-1990: Stellvertretender Vorsitzender des Zentralen Fachausschusses Entomologie der Gesellschaft für Natur und Umwelt im Kulturbund der DDR

- 1986-1990: Leiter des Arbeitskreises Hymenoptera des Zentralen Fachausschusses Entomologie der Gesellschaft für Natur und Umwelt im Kulturbund der DDR

- 1992-1993: 1. Stellvertreter des Vorsitzenden des NABU-Bundesfachausschusses Entomologie

- 1993 Mitglied des Landesfachausschusses Entomologie des NABU Landesverband Brandenburg e.V.

- 1993-2003: Vorsitzender des Vereins der Freunde und Förderer des Deutschen Entomologischen Instituts e.V. 
- 1995-2004: Mitglied/Vorsitzender des Naturschutzbeirates bei der unteren Naturschutzbehörde des Landkreises Barnim

- seit 2000: Vorstandsmitglied der Zentralen Arbeitsgemeinschaft Wirbellose im Terrarium e.V. (ZAG Wirbellose)

- 2003-2009: Mitglied/stellvertretender Vorsitzender des NABU-Kreisvorstandes Barnim

- seit 1992: Mitgliedschaft in Redaktionen oder wissenschaftlichen Beiräten von zoologischen Zeitschriften: Arthropoda. Zeitschrift der ZAG für Wirbellose im Terrarium, Berlin/ Roßlau (wissenschaftlicher Beirat 1993-1998); Beiträge zur Entomologie, Berlin/Keltern (Redaktionskollegium 1991; Chefredakteur 1992-1995; wissenschaftlicher Beirat seit 1996); Zoosystematica Rossica, St. Petersburg (International Advisory Board 1992-1996).

\section{Würdigungen und Auszeichnungen (in chronologischer Reihenfolge)}

- Dr.-Theodor-Neubauer-Medaille in Bronze (Juni 1964)

- Ehrennadel der Sportvereinigung Dynamo in Bronze (März 1973)

- Ehrennadel des Deutschen Turn- und Sportbundes der DDR (DTSB) in Bronze (1974)

- Ehrennadel der BSG Stahl Finow in Silber (Oktober 1978)

- Ehrennadel für besondere Leistungen im Naturschutz in Bronze (November 1982)

- Ehrennadel der Nationalen Front in Silber (Dezember 1985)

- Ehrenurkunde des Freien Deutschen Gewerkschaftsbundes (Juni 1984)

- Ehrenurkunde des Kulturbundes der DDR (Oktober 1984)

- Johannes-R.-Becher-Medaille des Kulturbundes der DDR (Juni 1986)

- Ehrennadel für besondere Leistungen im Naturschutz in Silber (September 1986)

- Ehrenmitglied der Naturforschenden Gesellschaft der Oberlausitz e.V. (März 1995)

- Ehrennadel des Naturschutzbund Deutschland e.V. (NABU) in Gold (April 2001)

- Freier Mitarbeiter des Deutschen Entomologischen Instituts (April 2001)

- Mitglied des Senckenberg Deutschen Entomologischen Instituts (Dezember 2007)

\section{Joachim Oehlke in den Printmedien (Auswahl)}

BAuER, A. 1985: Atlasspinner, Goldafter und die Honigbiene hinter Glas. Entomologische Lehr- und Anschauungsmaterialien werden in Eberswalde hergestellt. - Blick, 4. Januar 1985: S. 9; Chemnitz.

Bederke, J. 2000: Eberswalder Einblicke in die Welt der Sechsbeiner. - Berliner Morgenpost, 10. Januar 2000: S. 36; Berlin

Buchwald, R. 1993: Dr. rer. nat. Joachim Oehlke. - S. 166. - In: Skizzen und Porträts aus Eberwalde und Umgebung: 252 S.; Freiburg im Breisgau: Publicon-Verlags-GmbH.

DitTfeld, H. 2012: Tausende Heuschrecken, Käfer und auch Spinnen. Naturkundemuseum erhält umfangreiche Insektensammlung von früherem Professor / 40.000 Besucher im vergangenen Jahr. - Potsdamer Neueste Nachrichten, 13. Januar 2012: S. 8; Potsdam.

Loose, R. 2001: Wissenschaftler beginnt neuen Lebensabschnitt. Abschied aus vielen Jahren Berufsleben. Märkische Oderzeitung, 6. April 2001: S. 15; Frankfurt (Oder). 
Matthes, I. 2004: Steckbrief für die Cucaracha. Kakerlaken, Läuse und Wanzen - Ekel vor Ungeziefer ist den Eberswaldern Joachim und Renate OehlKe völlig fremd. Das Ehepaar gehört zu den wenigen Insektenpräparatoren in Deutschland. - Märkische Oderzeitung, 15.-16. Mai 2004. Wochenendjournal: S. 3; Frankfurt (Oder).

Redaktion Eberswalder Monatsblatt 1995: Prof. Dr. Dathe zum „50.“. - Eberswalder Monatsblatt, 1. August 1995: S. 4; Eberswalde.

Redaktion MOZ 1993: Einen herzlichen Empfang ... [sic!; ohne Titel]. - Märkische Oderzeitung, 30. November 1993: S. 11; Frankfurt (Oder).

Redaktion MOZ 1996a: Liebhaber tauschen Vogelspinnen. Ausstellung „Wirbellose im Terrarium“ im Entomologischen Institut. - Märkische Oderzeitung, 17. Oktober 1996: S. 7; Frankfurt (Oder).

Redaktion MOZ 1996b: Professor Dr. Joachim Oehlke (59). Der Schwarzgurt mit dem „Insektenfimmel“. - Märkische Oderzeitung, 18. Oktober 1996: S. 10; Frankfurt (Oder).

Redaktion MOZ 2001: Ehrennadel zum Kolloquium. - Märkische Oderzeitung, 11. April 2001: S. 15; Frankfurt (Oder).

Redaktion MOZ 2007: Professor OehlKe vererbt sein Werk. Thomas Kolling übernimmt Insektenkästen. - Märkische Oderzeitung, 6. März 2007: S. 13; Frankfurt (Oder).

Redaktion MOZ 2009: NABU mit neuem Chef. Mitglieder danken Professor Joachim Oenlke. Märkische Oderzeitung, 18. Juni 2009: S. 15; Frankfurt (Oder).

Reppen, H. 1994: Der ICE und der Käfer am Gleis. Bevorzugte Adresse für Insektenforscher: Projekt Entomologie, Fachhochschule Eberswalde. - Brandenburger Blätter Nr. 65, 7. Januar 1994: S. 9; Frankfurt (Oder).

Richter, I. 1991: Vom Atlasspinner bis zur Buckel-Zikade. Entomologisches Institut hat an die drei Millionen Exemplare „auf Lager“. - Deutsches Landblatt Nr. 51, 1. März 1991: S. 17; Berlin.

Schröder, C. 1992: Minister Enderlein, helfen Sie Endlich dem Insekten-Institut. - Berliner Kurier, 6. Dezember 1992: S. 6; Berlin.

Steinkraus, D. 1992: Insekten-Bibliothek noch ohne Zukunft. Übernahme der Forschungsanstalt dagegen gesichert; Arbeitsvertrag der Bibliothekarin nur bis 30. Juni. - Märkische Oderzeitung, 1. April 1992: S. 4; Frankfurt (Oder).

\section{Schriftenverzeichnis von Joachim Oehlke}

Das Schriftenverzeichnis erhebt keinen Anspruch auf Vollzähligkeit. In den Jahresberichten des Deutschen Entomologischen Institutes (AutorenKollektiv 1991-2009) lassen sich Vorworte und Tätigkeitsberichte von Joachim Oehlke finden, auf deren Zitation hier ausdrücklich verzichtet wurde. Außerdem können Forschungsberichte oder Artikel aus dem Bereich des Natur- und Umweltschutzes abgedruckt worden sein, von denen die Autoren keine Kenntnis haben. In dem nachfolgenden Schriftenverzeichnis wurden 119 Publikationen von Joachim Oehlke zusammengetragen.

Oenlke, J. 1962: Zur Larvalsystematik der Gattung Agriotes Esch. (Col. Elateridae). - Deutsche Entomologische Zeitschrift, Neue Folge 9 (3-4): 336-349; Berlin.

Oenlke, J. 1963: Ergebnisse der Albanien-Expedition 1961 des Deutschen Entomologischen Institutes. 15. Beitrag. Hymenoptera: Myrmosidae und Mutillidae. - Beiträge zur Entomologie 13 (7-8): 831-838; Berlin.

Oenlke, J. 1963: Der Fadenzieher. - Der Bäcker und Konditor. Fachmagazin für handwerkliche Bäcker und Konditoren in Berlin, Brandenburg, Mecklenburg-Vorpommern, Sachsen-Anhalt, Sachsen und Thüringen 11: 342-343; Alfeld. 
Oenlke, J. 1963: Revision der im Deutschen Entomologischen Institut aufbewahrten Typen paläarktischer Ichneumoniden (Hymenoptera, Ichneumonidae). - Beiträge zur Entomologie 13 (3-4): 403-410; Berlin.

Oenlke, J. 1964: Ergebnisse der Albanien-Expedition 1961 des Deutschen Entomologischen Institutes. 28. Beitrag. Hymenoptera: Ichneumonidae I (Ephialtinae). - Beiträge zur Entomologie 14 (3-4): 373-376; Berlin.

Oенцке, J. 1964: Übersicht und Bestimmungstabelle der palaearktischen Gattungen der ehemaligen Unterfamilie Pimplinae auct. (Hymenoptera, Ichneumonidae). - Entomologische Abhandlungen aus dem Staatlichen Museum für Tierkunde in Dresden 29 (10): 533-590; Dresden.

Oenlke, J. 1964: Hymenopteren als Parasiten der Schmetterlinge. - Entomologische Berichte 1963 (2): 81-82; Berlin.

Oenlke, J. 1965: Die westpalaearktischen Arten der Tribus Poemeniini (Hymenoptera: Ichneumonidae). Beiträge zur Entomologie 15 (7-8): 881-892; Berlin.

Oenlke, J. 1965: Beitrag zur Systematik und Faunistik des Genus Psen Latr. Subgenus Mimesa Shuck (Hym. Sphecidae). - Sborník entomologického oddělení Národního Musea v Praze [Acta Entomologica Musei Nationalis Pragae] 36: 339-347; Praha.

OenLKe, J. 1966: Die in europäischen Kiefernbuschhornblattwespen (Diprionidae) parasitierenden Ichneumonidae. - Beiträge zur Entomologie 15 (7-8) [1965]: 791-879; Berlin.

Oenlke, J. 1966: Apistephialtes Seyrig ein Synonym zu Liotryphon Ashmead (Hymenoptera, Ichneumonidae). - Reichenbachia 6 (18): 145-146; Leipzig.

Oenlke, J. 1966: Revision der Ephialtinen-Typen von A. Hensch (Hymenoptera, Ichneumonidae). Reichenbachia 6 (22): 175-180; Leipzig.

Oehlke, J. 1966: Revision der Ephialtinae-Typen von H. Haupt (Hymenoptera, Ichneumonidae). Reichenbachia 6 (32): 279-285; Leipzig.

Oenlke, J. 1966: Moderne taxonomische Forschung - Grundlage für Prognose und Methoden der Bekämpfung von Schadinsekten, dargestellt am Beispiel der Kiefernbuschhornblattwespen (Diprioninae) und ihrer Parasiten (Ichneumonidae). - Archiv für Forstwesen 15 (9): 953-958; Berlin.

Oenlke, J. 1966: Zur Synonymie einiger Scambus-Arten (Hymenoptera: Ichneumonidae). - Beiträge zur Entomologie 16 (1-2): 189-191; Berlin.

Oenlke, J. 1967: Westpaläarktische Ichneumonidae 1: Ephialtinae. - In: Ferrière, C. \& van der Vecht, J. (Hrsg.): Hymenopterorum Catalogus (nova editio) 2: 1-49; 's-Gravenhage: Dr. W. Junk.

Oehlke, J. 1967: Fang, Zucht und Präparation von Schlupfwespen (Hymenoptera, Ichneumonidae). - Entomologische Nachrichten 11 (6-7): 69-87; Dresden.

Oенцке, J. 1967: Ergebnisse der Albanien-Expedition 1961 des Deutschen Entomologischen Institutes. 66. Beitrag. Hymenoptera: Tiphiidae. - Beiträge zur Entomologie 17 (3-4): 517-520; Berlin.

OenLke, J. 1968: Zur Wegwespenfauna der Insel Hiddensee. Ein Beitrag zur Fauna von Naturschutzgebieten der DDR. (Hymenoptera: Pompiloidea). - Beiträge zur Entomologie 18 (3-4): 311-317; Berlin.

Oenlke, J. 1968: Über den Verbleib der Hymenopteren-Typen Schmiedeknechts. - Beiträge zur Entomologie 18 (3-4): 319-327; Berlin.

ČapeK, M.; Ǩ̌́́steK, J. \& OehlKe, J. 1969: Zur Problematik der Insektenparasiten des Fichtennestwicklers Epinotia tedella (CL.). - Acta Universitatis Agriculturae, řada C 38 (3): 271-283; Brno.

Oenlke, J. 1969: Beiträge zur Insektenfauna der DDR: Hymenoptera - Bestimmungstabellen bis zu den Unterfamilien. - Beiträge zur Entomologie 19 (7-8): 753-801; Berlin.

OenLKe, J. 1969: Zum Prinzip der Wirtswahl und Wirtsspezifität bei Ichneumoniden. - In: Bericht über die 10. Wanderversammlung Deutscher Entomologen, 15. bis 19. September 1965 in Dresden. - Tagungsberichte 80: 197-201; Berlin.

OenLKe, J. 1969: Hymenopterologische Arbeiten in Naturschutzgebieten. - Naturschutzarbeit in Mecklenburg 12 (2-3): 34-36; Schwerin.

Oenlke, J. \& Townes, H. K. 1969: Schmiedeknechts Ichneumonidentypen aus der Kollektion des Museums Rudolstadt. - Beiträge zur Entomologie 19 (3-6): 395-412; Berlin. 
Oenlke, J. 1970: Beiträge zur Insekten-Fauna der DDR: Hymenoptera - Sphecidae. - Beiträge zur Entomologie 20 (7-8): 615-812; Berlin.

Oehlke, J. 1971: Zur Situation der Ichneumonidenforschung in Europa. - S. 183. - In: Gureva, E. L. \& Kryshanovski, O. L. (Hrsg): XIII International Congress of Entomology. Moscow, 2-9 August, 1968. Proceedings, Volume 1: 581 S.; Leningrad: Nauka.

Oenlke, J. 1972: Zur Grabwespenfauna der Insel Hiddensee. Ein Beitrag zur Fauna von Naturschutzgebieten der DDR (Hymenoptera: Sphecidae). - Beiträge zur Entomologie 22 (3-6): 131-142; Berlin.

Oehlke, J. \& Wudowenz, J. 1973: Katalog der in den Sammlungen des ehemaligen Deutschen Entomologischen Institutes aufbewahrten Typen - X. Hymenoptera. Sphecidae. - Beiträge zur Entomologie 23 (5-8): 421-426; Berlin.

Muche, W. H. \& OenLKe, J. 1974: Zur Blattwespenfauna der Insel Hiddensee - II. Ein Beitrag zur Fauna von Naturschutzgebieten der DDR. (Hymenoptera: Symphyta). - Beiträge zur Entomologie 24 (5-8): 301-306; Berlin.

Oenlke, J. 1974: Beiträge zur Insektenfauna der DDR: Hymenoptera - Scolioidea. - Beiträge zur Entomologie 24 (5-8): 279-300; Berlin.

Oehlke, J. \& Dylewska, M. 1976: Zur Bienenfauna der Insel Hiddensee. Ein Beitrag zur Fauna von Naturschutzgebieten der DDR (Hymenoptera, Apoidea). - Beiträge zur Entomologie 25 (1): 39-48; Berlin.

Oenlke, J. 1978: Hymenoptera - Hautflügler. - S. 398-463. - In: Stresemann, E.; Senglaub, K. \& Hannemann, H.-J. (Hrsg.): Stresemann, Exkursionsfauna für die Gebiete der DDR und der BRD. Band 2.1, Wirbellose. Insekten - Erster Teil. - 4. stark bearbeitete Auflage, 504 S.; Berlin: Volk und Wissen Volkseigener Verlag.

Oenlke, J. 1978: Strepsiptera - Fächerflügler, Kolbenflügler. - S. 393-397. - In: Stresemann, E.; Senglaub, K. \& Hannemann, H.-J. (Hrsg.): Stresemann, Exkursionsfauna für die Gebiete der DDR und der BRD. Band 2.1, Wirbellose. Insekten - Erster Teil. - 4. stark bearbeitete Auflage, 504 S.; Berlin: Volk und Wissen Volkseigener Verlag.

OenLKe, J. 1979:Zur Faltenwespenfauna der Insel Hiddensee. Ein Beitrag zur Fauna von Naturschutzgebieten der DDR. (Hymenoptera, Vespoidea). - Beiträge zur Entomologie 29 (1): 289-293; Berlin.

Oenlke, J. 1980: Mitteilung des Zentralen Fachausschusses Entomologie. 2. Arbeitstagung der Hymenopterologen der DDR. - Entomologische Nachrichten 24 (9): 141-142; Dresden.

Oenlke, J. 1981: Zu entomologischen Sammelergebnissen im Bezirk Cottbus (Hymenoptera). - Biologische Studien im Kreis Luckau 10: 58-59; Luckau.

Oenlke, J. 1981: Hymenoptera - Hautflügler. - S. 398-463. - In: Stresemann, E.; Senglaub, K. \& Hannemann, H.-J. (Hrsg.): Stresemann, Exkursionsfauna für die Gebiete der DDR und der BRD. Band 2.1, Wirbellose. Insekten - Erster Teil. - 5. durchgesehene Auflage, 504 S.; Berlin: Volk und Wissen Volkseigener Verlag.

Oenlke, J. 1981: Strepsiptera - Fächerflügler, Kolbenflügler. - S. 393-397. - In: Stresemann, E.; Senglaub, K. \& Hannemann, H.-J. (Hrsg.): Stresemann, Exkursionsfauna für die Gebiete der DDR und der BRD. Band 2.1, Wirbellose. Insekten - Erster Teil. - 5. durchgesehene Auflage, 504 S.; Berlin: Volk und Wissen Volkseigener Verlag.

Oenlke, J. 1982: Zur Unterscheidung der Arten Mimesa caucasica Maidl und tenuis OehlKe (Hym. Sphecidae, Psenini). - Entomologische Nachrichten und Berichte 26 (2): 57-58; Leipzig.

OehlKe, J. 1982: Dr. sc. nat. Eberhard Königsmann (1930-1980). - Entomologische Nachrichten und Berichte 26 (1): 46-47; Leipzig.

Papp, J. \& Oenlke, J. 1982: Zur Brackwespenfauna der Insel Hiddensee. Ein Beitrag zur Fauna von Naturschutzgebieten der DDR. (Insecta, Hymenoptera, Braconidae). - Faunistische Abhandlungen. Staatliches Museum für Tierkunde in Dresden 9 (17): 185-193; Dresden.

Oenlke, J. 1983: Revision der europäischen Aulacidae (Hymenoptera - Evanioidea). - Beiträge zur Entomologie 33 (2): 439-447; Berlin. 
Oenlke, J. 1983: Zur Nomenklatur der Gattungen Trigonalis, Stephanus und Brachygaster (Hymenoptera, Trigonalyoidea, Stephanoidea et Evanioidea). - Reichenbachia 21 (14): 91-93; Leipzig.

Oenlke, J. 1983: Prof. Dr. Ulrich Sedlag 60 Jahre. - Entomologische Nachrichten und Berichte 27 (6): 281; Leipzig.

Oenlke, J. 1984: Beiträge zur Insektenfauna der DDR: Hymenoptera - Evanioidea, Stephanoidea, Trigonalyoidea (Insecta). - Faunistische Abhandlungen. Staatliches Museum für Tierkunde in Dresden 11 (13): 161-190; Dresden.

Oenlke, J. 1984: Hymenoptera - Hautflügler. - S. 398-463. - In: Stresemann, E.; Senglaub, K. \& Hannemann, H.-J. (Hrsg.): Stresemann, Exkursionsfauna für die Gebiete der DDR und der BRD. Band 2.1, Wirbellose. Insekten - Erster Teil. - 6. Auflage, 504 S.; Berlin: Volk und Wissen Volkseigener Verlag.

OenlKe, J. 1984: Strepsiptera - Fächerflügler, Kolbenflügler. - S. 393-397. - In: Stresemann, E.; Senglaub, K. \& Hannemann, H.-J. (Hrsg.): Stresemann, Exkursionsfauna für die Gebiete der DDR und der BRD. Band 2.1, Wirbellose. Insekten - Erster Teil. - 6. Auflage, 504 S.; Berlin: Volk und Wissen Volkseigener Verlag.

Oenlke, J. \& Szabó, J. B. 1984: Zur Kenntnis der in Getreidewanzeneiern obstanten Mikrohymenopteren (Proctotrupoidea: Scelionidae und Chalcidoidea: Encyrtidae) und zu Proctotrupoidea-Arten aus der Sammlung der Abteilung für Taxonomie der Insekten, Institut für Pflanzenschutzforschung Eberswalde (ehemals DEI). (Insecta, Hymenoptera). - Faunistische Abhandlungen. Staatliches Museum für Tierkunde in Dresden 11 (7) [1983]: 125-131; Dresden.

Oenlke, J. \& Wudowenz, J. 1984: Katalog der in den Sammlungen der Abteilung Taxonomie der Insekten des Institutes für Pflanzenschutzforschung, Bereich Eberswalde (ehemals Deutsches Entomologisches Institut), aufbewahrten Typen - XXII (Hymenoptera: Symphyta). - Beiträge zur Entomologie 34 (2): 363-420; Berlin.

OenLKe, J. 1986: Naturschutz und entomologisches Sammeln. - Entomologische Nachrichten und Berichte 30 (5): 227-235; Leipzig.

Oentke, J. 1986: Seen, Moore und Fließgewässer im Blickfeld unserer Aufmerksamkeit. - Unser Forum. Kulturinformationen für den Bezirk Frankfurt (Oder) 4: 9-10; Frankfurt (Oder): Bezirkskabinett für Kulturarbeit.

Oehlke, J. 1986: Hymenoptera - Hautflügler. - S. 398-463. - In: Stresemann, E.; Hannemann, H.-J.; Klausnitzer, B. \& Senglaub, K. (Hrsg.): Stresemann, Exkursionsfauna für die Gebiete der DDR und der BRD. Band 2.1, Wirbellose. Insekten - Erster Teil. - 7. Auflage, 504 S.; Berlin: Volk und Wissen Volkseigener Verlag.

Oenllke, J. 1986: Strepsiptera - Fächerflügler, Kolbenflügler. - S. 393-397. - In: Stresemann, E.; Hannemann, H.-J.; Klausnitzer, B. \& Senglaub, K. (Hrsg.): Stresemann, Exkursionsfauna für die Gebiete der DDR und der BRD. Band 2.1, Wirbellose. Insekten - Erster Teil. - 7. Auflage, 504 S.; Berlin: Volk und Wissen Volkseigener Verlag.

Szabó, J. \& Oehlke, J. 1986: Neue Proctotrupoidea aus dem Baltischen Bernstein. - Beiträge zur Entomologie 36 (1): 99-106; Berlin.

Listner, K. \& OeHLKe, J. 1987: Erfahrungen im langjährigen Biotopschutz an einem Fließgewässer. Auszüge aus dem Eröffnungsvortrag der Arbeitstagung der Ichthyofaunisten in Eberswalde. - Ichthyofaunistik 1987: 17-20; Rostock.

Oehlke, J. \& Horstmann, K. 1987: Die Hymenopterensammlung C. F. Lange/Annaberg und Revision seiner Ichneumoniden-Typen. - Beiträge zur Entomologie 37 (1): 147-157; Berlin.

Oenlke, J. \& Wolf, H. 1987: Beiträge zur Insekten-Fauna der DDR: Hymenoptera - Pompilidae. Beiträge zur Entomologie 37 (2): 279-390; Berlin.

Mey, E. \& Oehlke, J. 1988: Die Hymenopteren-Kollektion Otto Schmiedernechts im Naturhistorischen Museum Rudolstadt/Thür. - Rudolstädter Naturhistorische Schriften 1: 56-71; Rudolstadt. 
Oenlke, J. 1988: Anforderungen des Fachwissenschaftlers an den entomologischen Präparator. - Neue Museumskunde. Theorie und Praxis der Museumsarbeit 31 (1): 54-56; Berlin.

Oenlke, J. \& Geissler, I. 1988: Transkaukasien-Exkursion im Herbst 1987. - Mitteilungen der biologischen Gesellschaft der DDR 1988 (1): 43-47; Berlin.

Oehlke, J. \& Schneider, M. 1988: Max FüGe - am 3.12.1988 80 Jahre. - Entomologische Nachrichten und Berichte 32 (6): 282-283; Leipzig.

Oehlke, J. 1989: Hymenoptera - Hautflügler. - S. 398-463. - In: Stresemann, E.; Hannemann, H.-J.; Klausnitzer, B. \& Senglaub, K. (Hrsg.): Stresemann, Exkursionsfauna für die Gebiete der DDR und der BRD. Band 2.1, Wirbellose. Insekten - Erster Teil. - 8. Auflage, 504 S.; Berlin: Volk und Wissen Volkseigener Verlag.

Oehlke, J. 1989: Strepsiptera - Fächerflügler, Kolbenflügler. - S. 393-397. - In: Stresemann, E.; Hannemann, H.-J.; Klausnitzer, B. \& Senglaub, K. (Hrsg.): Stresemann, Exkursionsfauna für die Gebiete der DDR und der BRD. Band 2.1, Wirbellose. Insekten - Erster Teil. - 8. Auflage, 504 S.; Berlin: Volk und Wissen Volkseigener Verlag.

Oenlke, J. 1989: Hymenopterologische Eindrücke in der Mongolei. - Rundschreiben der Gesellschaft für Natur und Umwelt im Kulturbund der DDR, ZFA Entomologie, Arbeitskreis Hymenoptera 5: 4-5; Eberswalde: Selbstverlag.

Oenlke, J. 1989: Hymenopterologisches FND. - Rundschreiben der Gesellschaft für Natur und Umwelt im Kulturbund der DDR, ZFA Entomologie, Arbeitskreis Hymenoptera 5: 10-11; Eberswalde: Selbstverlag.

Oehlke, J. \& OehlKe, R. 1989: Bei Hymenopterologen zu Gast in Übersee. - Rundschreiben der Gesellschaft für Natur und Umwelt im Kulturbund der DDR, ZFA Entomologie, Arbeitskreis Hymenoptera 5: 6-7; Eberswalde: Selbstverlag.

Oenlke, J. \& Oenlke, R. 1989: Zur chemischen Präparation von Larvalstadien, insbesondere xylobionter Käferlarven. - Der Präparator 35 (1): 11-15; Bochum.

Oenlke, J. \& Sedlag, U. 1989: Zu einigen Aspekten des Biotop- und Artenschutzes. - Entomologische Nachrichten und Berichte 33 (5): 205-211; Leipzig.

Jacobs, H.-J. \& OehlKe, J. 1990: Beiträge zur Insektenfauna der DDR: Hymenoptera, Sphecidae. 1. Nachtrag. - Beiträge zur Entomologie 40 (1): 121-229; Berlin.

Oенцке, J. 1990: Zu einigen theoretischen Grundlagen des Schutzes bedrohter Insekten. - Entomologische Nachrichten und Berichte 34 (2): 49-56; Leipzig.

Oenlke, J. 1991: AK Hymenoptera. - Entomologische Nachrichten und Berichte 35 (2):108 ; Leipzig.

Oenlke, J. 1991: Univ. Prof. Dr. Hubert Pschorn-Walcher zu seinem 65. Geburtstag und zu seiner Emeritierung als o. Prof. für Ökologie in Kiel. - Entomologische Nachrichten und Berichte 35 (4): 281-282; Dresden.

Oenlke, J. \& Sacher, P. 1991: Speziation bei Parasitoiden am Beispiel von Schlupfwespen (Ichneumonidae: Pimplinae). - Mitteilungen aus dem Zoologischen Museum in Berlin 67 (1): 169-176; Berlin.

OehlKe, J. 1992: Museologie und entomologische Forschung. - Insecta 1 (1): 32-37; Berlin.

Oenlke, J. 1992: Arbeitsgruppe Hymenoptera, Diptera und übrige Insektengruppen. - Insecta 1 (1): 77-78; Berlin.

Oehlke, J. 1992: Schriftenverzeichnis von H. Pschorn-Walcher. - Entomologische Nachrichten und Berichte 36 (1): 68-70; Dresden.

Oenlke, J. 1992: Bericht zur hymenopterologischen Arbeitstagung in Eberswalde im September 1991. DGaaE-Nachrichten 6 (2): 46-48; Ulm.

Oenlke, J. 1992: Zur Weiterführung und Erweiterung der „Fauna der DDR“. - S. 554-559. - In: Zombori, L. \& Peregovits, L. (Hrsg): Proceedings of the Fourth European Congress of Entomology and the XIII. Internationale Symposium für die Entomofaunistik Mitteleuropas, 1-6 September, 1991, Gödöllő, Hungary. Volume 2: 884 S.; Budapest: Hungarian Natural History Museum. 
OeHLKe, J. 1992: Insektenkunde im Naturschutzbund. - Ökowerkmagazin 6 (10): 21; Berlin.

Oenlke, J. (Koord.) 1992: Rote Liste. Hautflügler (Hymenoptera). - S. 63-96. - In: Ministerium für Umwelt, Naturschutz und Raumordnung des Landes Brandenburg (Hrsg.): Rote Liste. Gefährdete Tiere im Land Brandenburg: 288 S.; Potsdam: Unze Verlagsgesellschaft mbH.

OenLKe, J. 1992: Faltenwespen (Vespoidea). - S. 71-72. - In: Ministerium für Umwelt, Naturschutz und Raumordnung des Landes Brandenburg (Hrsg.): Rote Liste. Gefährdete Tiere im Land Brandenburg: 288 S.; Potsdam: Unze Verlagsgesellschaft mbH.

OenlKe, J. 1992: Wegwespen (Pompilidae) und Schmarotzerwespen (Ceropalidae). - S. 73-74. - In: Ministerium für Umwelt, Naturschutz und Raumordnung des Landes Brandenburg (Hrsg.): Rote Liste. Gefährdete Tiere im Land Brandenburg: 288 S.; Potsdam: Unze Verlagsgesellschaft mbH.

Oenlke, J. 1992: Grabwespen (Sphecidae). - S. 75-79. - In: Ministerium für Umwelt, Naturschutz und Raumordnung des Landes Brandenburg (Hrsg.): Rote Liste. Gefährdete Tiere im Land Brandenburg: 288 S.; Potsdam: Unze Verlagsgesellschaft mbH.

OenLKe, J. 1992: Dolch-, Roll- und Keulenwespen sowie Bienen- oder Trugameisen (Scolioidea). - S. 80-81. - In: Ministerium für Umwelt, Naturschutz und Raumordnung des Landes Brandenburg (Hrsg.): Rote Liste. Gefährdete Tiere im Land Brandenburg: 288 S.; Potsdam: Unze Verlagsgesellschaft mbH.

Oenlke, J. 1992: Hungerwespen (Evaniidae), Schmalbauchwespen (Gasteruptiidae) sowie Aulacidae und Trigonalyoidae. - S. 82. - In: Ministerium für Umwelt, Naturschutz und Raumordnung des Landes Brandenburg (Hrsg.): Rote Liste. Gefährdete Tiere im Land Brandenburg: 288 S.; Potsdam: Unze Verlagsgesellschaft mbH.

OenLKe, J. 1992: Schlupfwespen, Goldwespen und weitere paraitoide [sic!] Hautflüglergruppen (Ichneumonidea [sic!], Cynipoidea, Bethyloidea). - S. 83-84. - In: Ministerium für Umwelt, Naturschutz und Raumordnung des Landes Brandenburg (Hrsg.): Rote Liste. Gefährdete Tiere im Land Brandenburg: 288 S.; Potsdam: Unze Verlagsgesellschaft mbH.

Dathe, H. H. \& Oehlкe, J. 1993: Zum Problem Lindenblüte und Insektensterben. - Insecta 1 (2): 161-165; Berlin.

Kudrna, O.; Oehlke, J.; von Knorre, D.; Kühling, M. \& Sprick, P. 1993: Ehrencodex der Entomologischen Feldarbeit. - Mitteilungsblatt des BFA Entomologie im Naturschutzbund 1993 (2): 1-3; Berlin.

OehlKe, J. 1993: Die Schwärze und das Nonnenfließ. - S. 120-122. - In: Verein für Heimatkunde zu Eberswalde e.V. (Hrsg.): Eberswalder Jahrbuch für Heimat, Kultur- und Naturgeschichte 1993. 136 S.; Eberswalde: Selbstverlag.

OenlKe, J. \& Grönke, O. 1993: Eine biologische Expedition in Nicaragua (Teil 1). - Partner. Journal für Mitglieder und Freunde der BBAG 2 (3): 18; Potsdam.

Oehlke, J. \& Petersen, G. 1993: Zur Tradition der Wanderversammlungen Deutscher Entomologen. Geschichte, Zielstellungen, Ergebnisse, Perspektive. - Mitteilungen der Deutschen Gesellschaft für allgemeine und angewandte Entomologie 8 (4-6): 875-878; Giessen.

Oehlke, J. 1994: Hymenoptera - Hautflügler. - S. 398-463. - In: Hannemann, H.-J. \& Klausnitzer, B. (Hrsg.): Stresemann, Exkursionsfauna von Deutschland. Band 2.1, Wirbellose. Insekten - Erster Teil. 8. Auflage (Neuausgabe), 504 S.; Jena: Gustav Fischer Verlag.

Oenlke, J. 1994: Strepsiptera - Fächerflügler, Kolbenflügler. - S. 393-397. - In: Hannemann, H.-J. \& Klausnitzer, B. (Hrsg.): Stresemann, Exkursionsfauna von Deutschland. Band 2.1, Wirbellose. Insekten - Erster Teil. - 8. Auflage (Neuausgabe), 504 S.; Jena: Gustav Fischer Verlag.

OenlKe, J. 1995: Karl Bleyl. *04.12.1908 †28.04.1995. - Beiträge zur Entomologie 45 (2): 463-464; Berlin.

Oenlke, J.; von Broen, B. \& Burger, F. 1996: Zoologische Leitarten und Zielarten der bedeutsamsten Biotoptypen des Bundeslandes Brandenburg - Teil Wirbellose Tiere. Endbericht 1996. 220 S.; Eberswalde: Fachhochschule Eberswalde, Fachbereich Landschaftsnutzung und Naturschutz [unveröffentlicht]. 
Oehlke, J.; Burger, F. \& von Broen, B. 1997: Wirbellose als Leit- und Zielarten in den Fließgewässern Brandenburgs. - 39-50. - In: NABU Landesverband Brandenburg e.V. (Hrsg.): Wasser-Lebensraum für Pflanzen und Tiere. Fließgewässer in Brandenburg. Tagungsberichte vom 3. Naturschutztag des NABU Landesverbandes Brandenburg am 27. September 1997 in Potsdam. 80 S.; Berlin: movimento network.

Oehlke, J.; von Broen, B.; Burger, F. 1997: Praxiserprobung des Leit- und Zielartenschlüssels wirbelloser Tiere für die naturschutzfachliche Bewertung ausgewählter Standorte (Biotope) Brandenburgs (und Berlins). Endbericht 1997, 62 S. [incl. Anlage 1 und 2]; Eberswalde: Fachhochschule Eberswalde, Fachbereich Landschaftsnutzung und Naturschutz [unveröffentlicht].

Burger, F.; Saure, C. \& Oenleke, J. 1998 [unter Mitarbeit von Hinrichsen, A.; Jacobs, H.-J. \& Liebig, W.-H.]: Rote Liste und Artenliste der Grabwespen und weiterer Hautflüglergruppen des Landes Brandenburg (Hymenoptera: Sphecidae, Vespoidea part., Evanioidea, Trigonalyoidea). - Naturschutz und Landschaftspflege in Brandenburg, Beilage zu 7 (2): 24-43; Potsdam.

Oentke, J. 1998: Biomonitoring auf der Grundlage von Leit- und Zielarten wirbelloser Tiere. - S. 53-57. In: VAhrson, W.-G. \& Goldschmidt, B. (Hrsg.): Naturschutzbezogenes Monitoring von Landschaften. - Eberswalder wissenschaftliche Schriften 2: 1-165; Berlin: Deutscher Landwirtschaftsverlag.

Saure, C.; Burger, F. \& Oehleke, J. 1998 [unter Mitarbeit von Hinrichsen, A.; Liebig, W.-H. \& Nienuis, O.]: Rote Liste und Artenliste der Gold-, Falten- und Wegwespen des Landes Brandenburg (Hymenoptera: Chrysididae, Vespidae, Pompilidae). - Naturschutz und Landschaftspflege in Brandenburg, Beilage zu 7 (2): 3-23; Potsdam.

Eisermann, K.; Schulz, U. \& Oehlke, J. 2000: Die Libellenfauna (Odonata) eines extensiv genutzten Schiffahrtkanals: der Finowkanal in Eberswalde. - Entomologische Nachrichten und Berichte 44 (4): 253-258; Dresden.

Oenlke, J. 2000: Leit- und Zielarten in der Naturschutzarbeit Brandenburgs. - S. 5-10. - In: Osten, T. (Hrsg.): Beiträge der Hymenopterologen-Tagung in Stuttgart (6.-8.10.2000). 73 S.; Stuttgart: Staatliches Museum für Naturkunde Stuttgart.

Oenlke, J. 2000 [unter Mitarbeit von Taeger, A.; Blank, S. M.; Kraus, M; Dathe, H. H. \& Jacobs, H.-J.]: Hymenoptera - Hautflügler. - S. 803-912. - In: Hannemann, H.-J.; Klausnitzer, B. \& Senglaub, K. (Hrsg.): Stresemann, Exkursionsfauna von Deutschland, Band 2. Wirbellose: Insekten. 9. neu bearbeitete Auflage, 959 S.; Heidelberg \& Berlin: Spektrum Akademischer Verlag.

Oenlke, J. 2000: Strepsiptera - Fächerflügler, Kolbenflügler. - S. 696-701. - In: Hannemann, H.-J.; Klausnitzer, B. \& Senglaub, K. (Hrsg.): Stresemann, Exkursionsfauna von Deutschland, Band 2. Wirbellose: Insekten. 9. neu bearbeitete Auflage, 959 S.; Heidelberg \& Berlin: Spektrum Akademischer Verlag.

Oehleke, J. 2001: Vespidae. - S. 129-133. - In: Dathe, H. H.; Taeger, A. \& Blank, S. M. (Hrsg.): Verzeichnis der Hautflügler Deutschlands (Fauna Germanica 4). - Entomologische Nachrichten und Berichte, Beiheft 7: 178 S.; Dresden.

Oenlke, J. 2001: Pompilidae. - S. 133-136. - In: Dathe, H. H.; Taeger, A. \& Blank, S. M. (Hrsg.): Verzeichnis der Hautflügler Deutschlands (Fauna Germanica 4). - Entomologische Nachrichten und Berichte, Beiheft 7: 178 S.; Dresden.

Poht, H. \& Oenlкe, J. 2003: Verzeichnis der Fächerflügler (Strepsiptera) Deutschlands. - S. 273-275. In: Klausnitzer, B. (Hrsg.): Entomofauna Germanica 6. - Entomologische Nachrichten und Berichte, Beiheft 8: 344 S.; Dresden.

Oentke, J. 2004: DEI. Verlagerung war falsch. - Märkische Oderzeitung, 16. Juni 2004: S. 19; Frankfurt (Oder).

Börner, A.; Endtmann, K. J.; Oehlke, J. \& Möller, J. 2004: 5.2 Südlich Spechthausen. Nonnenfließtal. S. 204-215. - In: Schroeder, J. H. (Hrsg.): Nordwestlicher Barnim - Eberswalder Urstromtal. - Führer zur Geologie von Berlin und Brandenburg, Band 5: 313 S.; Berlin: Selbstverlag, Geowissenschaftler in Berlin und Brandenburg e. V. 
Oenlke, J. 2005 [unter Mitarbeit von Taeger, A.; Blank, S. M.; Kraus, M; Dathe, H. H. \& Jacobs, H.-J.]: Hymenoptera - Hautflügler. - S. 803-912. - In: Klausnitzer, B.; Hannemann, H.-J. \& Senglaub, K. (Hrsg.): Stresemann, Exkursionfauna von Deutschland, Band 2. Wirbellose: Insekten. 10. durchgesehene Auflage, 959 S.; München: Elsevier, Spektrum Akademischer Verlag.

Oenlke, J. 2005: Strepsiptera - Fächerflügler, Kolbenflügler. - S. 696-701. - In: Klausnitzer, B.; Hannemann, H.-J. \& Senglaub, K. (Hrsg.): Stresemann, Exkursionfauna von Deutschland, Band 2. Wirbellose: Insekten. 10. durchgesehene Auflage, 959 S.; München: Elsevier, Spektrum Akademischer Verlag.

Oehlke, J. \& Menzel, F. 2006: Entomologen im Land der Lemuren. Allgemeiner Bericht über eine Studienreise nach Madagaskar. - Studia dipterologica 12 (2) (2005): 385-407; Halle (Saale).

OehlKe, J. 2007: Grußwort. - S. 9. - In: Ministerium für LäNdliche Entwicklung, Umwelt und Verbraucherschutz des Landes Brandenburg \& Landesforstanstalt Eberswalde (Hrsg.): 100 Jahre Naturschutzgebiet Plagefenn. Ein Beispiel für erfolgreiches Zusammenwirken von Forstwirtschaft und Naturschutz. - Tagungsband zur Jubiläumsveranstaltung vom 11.-12. Mai 2007 in Chorin. - Eberswalder Forstliche Schriftenreihe 31: 1-130; Eberswalde.

Oenlke, J. 2009: 1.8. Familie Oehlke in Französisch Guayana. - S. 45-48. - In: Autorenkollektiv: Deutsches Entomologisches Institut. Jahresbericht 2007/2008. 87 S.; Müncheberg: Selbstverlag.

Oenlke, J. 2011: Hymenoptera - Hautflügler [alles außer Symphyta, Apidae, Sphecidae, Crysididae]. S. 572-586, 641, 644-681. - In: Klausnitzer, B. (Hrsg.): Stresemann, Exkursionsfauna von Deutschland, Band 2. Wirbellose: Insekten. 11. neu bearbeitete und erweiterte Auflage, 976 S.; Heidelberg: Spektrum Akademischer Verlag.

Oenlke, J. 2011: Strepsiptera - Fächerflügler, Kolbenflügler. - S. 826-831. - In: Klausnitzer, B. (Hrsg.): Stresemann, Exkursionsfauna von Deutschland, Band 2. Wirbellose: Insekten. 11. neu bearbeitete und erweiterte Auflage, 976 S.; Heidelberg: Spektrum Akademischer Verlag.

Ssymank, A.; Menzel, F.; Oenlke, J. \& Achukwi, D. 2011: Kamerun 2006 - Allgemeiner Bericht über eine entomologische Expedition nach Zentralafrika. - Studia dipterologica 17 (1-2) (2010): 267-316; Müncheberg.

OenLKe, J. 2012: Beitrag zur Kenntnis der Faltenwespen-Fauna von Kyrgyzstan (Hymenoptera: Vespidae \& Eumenidae). - Linzer biologische Beiträge 44 (1): 595-600; Linz.

\section{Danksagung}

Die Autoren bedanken sich recht herzlich bei Arne Köhler, Gabriele Mirschel und Renate Riedelsheimer (SDEI Müncheberg) für die Bereitstellung von Zeitungsartikeln und Literaturquellen sowie für ihre Recherchen im Archiv und in der Bibliothek des Senckenberg Deutschen Entomologischen Institutes. Helga Wehrens stellte weitere Bilder zur Verfügung.

\section{Literatur}

Autorenkollektiv 1991: Jahresbericht 1990. Biologische Zentralanstalt Berlin. 60 S.; Braunschweig: Biologische Bundesanstalt.

Autorenkollektiv 1992: Jahresbericht 1991. Biologische Zentralanstalt Berlin. 66 S.; Braunschweig: Biologische Bundesanstalt.

Autorenkollektiv 1993: Jahresbericht 1992 der Projektgruppe Entomologie (ehem. Deutsches Entomologisches Institut). 41 S.; Eberswalde: Selbstverlag.

Autorenkollektiv 1994: Projektgruppe Entomologie (ehem. Deutsches Entomologisches Institut). Jahresbericht 1993. 40 S.; Eberswalde: Selbstverlag. 
Autorenkollektiv 1995: Jahresbericht 1994. „Deutsches Entomologisches Institut“. Projektgruppe in der Fachhochschule Eberswalde. 52 S.; Eberswalde: Selbstverlag.

Autorenkollektiv 1996: Jahresbericht 1995. „Deutsches Entomologisches Institut“. Projektgruppe in der Fachhochschule Eberswalde. 52 S.; Eberswalde: Selbstverlag.

Autorenkollektiv 1997: Jahresbericht 1996. „Deutsches Entomologisches Institut“. Projektgruppe in der Fachhochschule Eberswalde. 68 S.; Eberswalde: Selbstverlag.

Autorenkollektiv 1998: Jahresbericht 1997. „Deutsches Entomologisches Institut“. 62 S.; Eberswalde: Selbstverlag.

Autorenkollektiv 1999: Jahresbericht 1998. „Deutsches Entomologisches Institut“. 62 S.; Eberswalde: Selbstverlag.

Autorenkollektiv 2000: Jahresbericht 1999. „Deutsches Entomologisches Institut“. 54 S.; Eberswalde: Selbstverlag.

Autorenkollektiv 2003: Jahresbericht 2000/2001. Deutsches Entomologisches Institut. 80 S.; Eberswalde: Selbstverlag.

Autorenkollektiv 2004: Jahresbericht 2002/2003 für das Deutsche Entomologische Institut. 66 S.; Müncheberg: Selbstverlag.

Autorenkollektiv 2006: Jahresbericht 2004 für das Deutsche Entomologische Institut. 48 S.; Müncheberg: Selbstverlag.

Autorenkollektiv 2007: Jahresbericht 2005/2006 für das Deutsche Entomologische Institut. 76 S.; Müncheberg: Selbstverlag.

Autorenkollektiv 2009: Deutsches Entomologisches Institut. Jahresbericht 2007/2008. 87 S.; Müncheberg: Selbstverlag.

Autorenkollektiv 2010: Senckenberg Deutsches Entomologisches Institut. Jahresbericht 2009. 92 S.; Müncheberg: Selbstverlag.

Bederke, J. 2001: Keine Krabbler mehr in Eberswalde. Größte ostdeutsche Insektenausstellung des Deutschen Entomologischen Institutes ist dicht. - Märkischer Markt Nr. 41/01, 10.-11. Oktober 2001. S. 6; Frankfurt (Oder).

Dathe, H. H. 2010: Studien zur Systematik und Taxonomie der Gattung Hylaeus F. (6). Arten asiatischer Hochgebirge und Anmerkungen zu weiteren asiatischen Arten (Hymenoptera, Anthophila, Colletidae). - Linzer Biologische Beiträge 42 (1): 43-80; Linz.

DitTfeld, H. 2012: Tausende Heuschrecken, Käfer und auch Spinnen. Naturkundemuseum erhält umfangreiche Insektensammlung von früherem Professor / 40.000 Besucher im vergangenen Jahr. - Potsdamer Neueste Nachrichten, 13. Januar 2012. S. 8; Potsdam.

Gusenleitner, J. 2008: Eine neue Stenodynerus-Art aus Kirgisien (Hymenoptera: Vespidae, Eumeninae). Linzer Biologische Beiträge 40 (2): 1491-1493; Linz.

Heinrich, G. H. 1970: Zur Systematik der Ichneumoninae Stenopneusticae. IX. Eine Spätlese (Hymenoptera, Ichneumonidae). - Mitteilungen der Münchner Entomologischen Gesellschaft 60: 80-101; München.

МомоI, S. 1973: Ergebnisse der zoologischen Forschungen von Dr. Z. KasZaB in der Mongolei. 331. Einige mongolischen [sic!] Arten der Unterfamilien Ephialtinae und Xoridinae (Hymenoptera: Ichneumonidae). - Folia Entomologica Hungarica (series nova) 26 (Supplement): 219-239; Budapest.

Muche, W. H. 1973: Die Blattwespen meiner Mittelasienausbeute 1972. - Entomologische Nachrichten 17 (11-12): 161-170; Dresden.

Papp, J. \& Oenlke, J. 1982: Zur Brackwespenfauna der Insel Hiddensee. Ein Beitrag zur Fauna von Naturschutzgebieten der DDR. (Insecta, Hymenoptera, Braconidae). - Faunistische Abhandlungen. Staatliches Museum für Tierkunde in Dresden 9 (17): 185-193; Dresden.

Redaktion MOZ 2001: Ehrennadel zum Kolloquium. - Märkische Oderzeitung, 11. April 2001. S. 15; Frankfurt (Oder). 
Rohlfien, K. \& Kroel, D. 1995: Deutsches Entomologisches Institut. Kleiner Institutsführer: 98 S.; Eberswalde: Deutsches Entomologisches Institut.

TAeger, A. 1989: Die Orgilus-Arten der Paläarktis (Hymenoptera, Braconidae). 260 S.; Berlin (1988): Akademie der Landwirtschaftswissenschaften der Deutschen Demokratischen Republik.

Zerche, L. 1990: Monographie der paläarktischen Coryphiini (Coleoptera, Staphylinidae, Omaliinae). 413 S.; Berlin: Akademie der Landwirtschaftswissenschaften der Deutschen Demokratischen Republik.

\section{Anschriften der Autoren}

Dr. Frank Menzel

Dr. Andreas Taeger

Dr. Stephan M. Blank

frank.menzel@senckenberg.de andreas.taeger@senckenberg.de

Christian Kutzscher stephan.blank@senckenberg.de

Lutz Behne christian.kutzscher@senckenberg.de lutz.behne@senckenberg.de

Senckenberg Deutsches Entomologisches Institut

Eberswalder Straße 90

15374 Müncheberg

Deutschland

Ortrud TAeger

Waldstraße 19

16225 Eberwalde

Deutschland

E-Mail: ortrud@taeger.at

Dr. Jens MölleR

Hochschule für Nachhaltige Entwicklung Eberswalde (FH)

Fachbereich Landschaftsnutzung und Naturschutz

Friedrich-Ebert-Straße 28

16225 Eberswalde

Deutschland

E-Mail: jens.moeller@hnee.de 